\title{
The High-Density lonized Gas in the Central Parsec of the Galaxy
}

\section{Citation}

Zhao, Jun-Hui, Raymond Blundell, James M. Moran, Dennis Downes, Karl F. Schuster, and Daniel P. Marrone. 2010. "The High-Density lonized Gas in the Central Parsec of the Galaxy." Astrophysical Journal 723 (2) (November 10): 1097-1109. doi:10.1088/0004-637X/723/2/1097.

\section{Published Version}

doi:10.1088/0004-637X/723/2/1097

\section{Permanent link}

http://nrs.harvard.edu/urn-3:HUL.InstRepos:11595847

\section{Terms of Use}

This article was downloaded from Harvard University's DASH repository, and is made available under the terms and conditions applicable to Other Posted Material, as set forth at http:// nrs.harvard.edu/urn-3:HUL.InstRepos:dash.current.terms-of-use\#LAA

\section{Share Your Story}

The Harvard community has made this article openly available.

Please share how this access benefits you. Submit a story.

Accessibility 


\title{
THE HIGH-DENSITY IONIZED GAS IN THE CENTRAL PARSEC OF THE GALAXY
}

\author{
Jun-Hui ZhaO ${ }^{1}$, Ray Blundell ${ }^{1}$, James M. Moran $^{1}$, Dennis Downes ${ }^{2}$, Karl F. Schuster ${ }^{2}$, And Daniel P. Markone ${ }^{3,4}$ \\ ${ }^{1}$ Harvard-Smithsonian Center for Astrophysics, 60 Garden Street, MS 78, Cambridge, MA 02138, USA; jzhao@cfa.harvard.edu \\ ${ }^{2}$ Institut de Radio Astronomie Millimétrique, 38406 Saint Martin d'Hères, France \\ ${ }^{3}$ Department of Astronomy and Astrophysics, University of Chicago, Chicago, IL 60637, USA \\ Received 2010 May 18; accepted 2010 September 4; published 2010 October 19
}

\begin{abstract}
We report a study of the $\mathrm{H} 30 \alpha$ line emission at $1.3 \mathrm{~mm}$ from the region around $\mathrm{Sgr} \mathrm{A}^{*}$ made with the Submillimeter Array at a resolution of $2^{\prime \prime}$ over a field of $60^{\prime \prime}(2 \mathrm{pc})$ and a velocity range of -360 to $+345 \mathrm{~km} \mathrm{~s}^{-1}$. This field encompasses most of the Galactic center's "minispiral." With an isothermal homogeneous H II model, we determined the physical conditions of the ionized gas at specific locations in the Northern and Eastern Arms from the H30 $\alpha$ line data along with Very Large Array data from the $\mathrm{H} 92 \alpha$ line at $3.6 \mathrm{~cm}$ and from the radio continuum emission at $1.3 \mathrm{~cm}$. The typical electron density and kinetic temperature in the minispiral arms are $3-21 \times 10^{4} \mathrm{~cm}^{-3}$ and $5000-13,000 \mathrm{~K}$, respectively. The $\mathrm{H} 30 \alpha$ and $\mathrm{H} 92 \alpha$ line profiles are broadened due to the large velocity shear within and along the beam produced by dynamical motions in the strong gravitational field near Sgr A*. We constructed a threedimensional model of the minispiral using the orbital parameters derived under the assumptions that the gas flows are in Keplerian motion. The gas in the Eastern Arm appears to collide with the Northern Arm flow in the "Bar" region, which is located $0.1-0.2 \mathrm{pc}$ south of and behind Sgr A*. Finally, a total Lyman continuum flux of $3 \times 10^{50}$ photons $\mathrm{s}^{-1}$ is inferred from the assumption that the gas is photoionized and the ionizing photons for the high-density gas in the minispiral arms are from external sources, which is equivalent to $\sim 250$ O9-type zero-age-main-sequence stars.
\end{abstract}

Key words: Galaxy: center - H II regions - ISM: individual objects (Sgr A) - ISM: kinematics and dynamics radio lines: ISM

Online-only material: color figures

\section{INTRODUCTION}

The Galactic center harbors a supermassive black hole (SMBH) with a mass of about $4.2 \times 10^{6} M_{\odot}$ (Ghez et al. 2008; Gillessen et al. 2009) at the position of the radio source Sgr A*. The inner parsec of the Galactic center region contains a rich cluster of stars, including at least $55 \mathrm{OB}$ stars within the central $0.5 \mathrm{pc}$ (Paumard et al. 2006). There are several theories to explain how these stars got there. In situ formation, as in giant molecular clouds, is improbable because of the stars' large velocity dispersion. The accretion sequence may or may not include the formation of an intermediate mass black hole in a super-dense stellar cluster that stabilizes the cluster against tidal disruption (Hansen \& Milosavljevic 2003). The mystery of the formation of these stars is closely linked to the balance of mass inflow and outflow toward the Galactic center and its interaction with the source Sgr A*. Infall of interstellar material may lead to star formation up to a certain radius from the SMBH, and the subsequent feedback from stellar mass loss may suppress further star formation. Both phenomena may determine whether Sgr A* is in a quiescent state of the active galactic nuclei cycle (Loeb 2004).

Earlier radio recombination line (RRL) studies (Schwarz et al. 1989; Roberts \& Goss 1993; Roberts et al. 1996) revealed complex structures of ionized gas, including the "minispiral" and "Bar," whose dynamics remain unclear. Parts of these structures have been modeled as gas orbiting Sgr A* (Schwarz et al. 1989; Sanders 1998; Paumard et al. 2004; Vollmer \& Duschl 2000; Liszt 2003; Mužić et al. 2007; Zhao et al. 2009) while other parts of the structures may be gravitationally unbound (Yusef-Zadeh et al. 1998). Another possibility is that

\footnotetext{
${ }^{4}$ Hubble Fellow.
}

some of the nearby stellar clusters (IRS 13 and IRS 16) have powerful winds that, with the help of gravitational focusing, create large-scale, unbound flows (e.g., Lutz et al. 1993). There are a number of limitations in the existing spectral line observations of these structures. In the centimeter-radio images made with the Very Large Array (VLA), ${ }^{5}$ the recombination line strength is low, the line-to-continuum ratio is low, and the bandwidth is limited, making high-velocity line wings especially difficult to detect. In the infrared, the picture given by the emission lines from the ionized gas is subject to corrections for dust extinction of $\sim 3 \mathrm{mag}$ in the central parsec (Scoville et al. 2003; Buchholz et al. 2009; Schödel et al. 2010). A few key questions about the ionized medium associated with Sgr A* are as follows.

1. Is the ionized material related to stars or star formation?

2. Is the ionized material streaming freely in the central gravitational potential, or do other mechanisms such as stellar winds and shocks influence the dynamics?

3. What are the column densities (or emission measures (EMs)), densities, kinetic temperatures, and dynamics of the H II gas in the vicinity of Sgr $\mathrm{A}^{*}$ itself?

In this paper, we present new observational results on the ionized gas in the central parsec of the Galaxy based on observations of the $\mathrm{H} 30 \alpha$ line made with the Submillimeter Array (SMA) ${ }^{6}$ and we interpret them in combination with

\footnotetext{
5 The Very Large Array (VLA) is operated by the National Radio Astronomy Observatory (NRAO). The NRAO is a facility of the National Science Foundation operated under cooperative agreement by Associated Universities, Inc.

6 The Submillimeter Array is a joint project between the Smithsonian Astrophysical Observatory and the Academia Sinica Institute of Astronomy and Astrophysics and is funded by the Smithsonian Institution and the Academia Sinica.
} 
Table 1

Summary of the $\mathrm{H} 30 \alpha$ Line Observations at $v=231.901 \mathrm{GHz}$

\begin{tabular}{|c|c|c|c|c|}
\hline Date (yyyy-mm-dd) & $\begin{array}{c}t_{\text {obs }} \text { on Sgr } \mathrm{A}^{*} \\
(\mathrm{hr})\end{array}$ & $\begin{array}{l}T_{\text {sys }} \\
(\mathrm{K}) \\
\end{array}$ & Array $^{\mathrm{a}}$ & Calibrators \\
\hline 2006-04-08 & 1.79 & 96 & $\mathrm{C}$ & $3 \mathrm{C} 273^{\mathrm{b}}$, Callisto $^{\mathrm{c}}$ \\
\hline $2006-05-28$ & 3.99 & 173 & $\mathrm{E}$ & $3 \mathrm{C} 279^{\mathrm{b}}$, Uranus $^{\mathrm{b}}, \mathrm{J} 1924-292^{\mathrm{b}}$, Callisto ${ }^{\mathrm{b}, \mathrm{c}}$ \\
\hline 2006-07-17 & 2.19 & 146 & $\mathrm{~V}$ & $3 \mathrm{C} 279^{\mathrm{b}}, \mathrm{J} 1924-292^{\mathrm{b}}$, Callisto $^{\mathrm{c}}$ \\
\hline $2007-03-31$ & 3.67 & 108 & $\mathrm{C}$ & $3 \mathrm{C} 273^{\mathrm{b}}$, Callisto $^{\mathrm{c}}$ \\
\hline 2007-04-01 & 3.35 & 126 & $\mathrm{C}$ & $3 \mathrm{C} 273^{\mathrm{b}}$, Callisto $^{\mathrm{c}}$ \\
\hline 2007-04-03 & 1.99 & 93 & $\mathrm{C}$ & $3 \mathrm{C} 273^{\mathrm{b}}$, Callisto $^{\mathrm{c}}$ \\
\hline 2007-04-04 & 1.93 & 103 & $\mathrm{C}$ & $3 \mathrm{C} 273^{\mathrm{b}}$, Callisto $^{\mathrm{c}}$ \\
\hline 2007-08-14 & 3.63 & 115 & $\mathrm{E}$ & $3 \mathrm{C} 279^{\mathrm{b}}$, NRAO $530^{\mathrm{c}}$ \\
\hline $2007-08-20$ & 4.94 & 95 & $\mathrm{E}$ & $3 \mathrm{C} 454.3^{\mathrm{b}}$, NRAO $530^{\mathrm{c}}$ \\
\hline $2007-08-31$ & 4.19 & 134 & $\mathrm{E}$ & $3 \mathrm{C} 454.3^{\mathrm{b}}$, NRAO $530^{\mathrm{c}}$ \\
\hline $2008-05-05$ & 2.67 & 156 & $\mathrm{C}$ & $3 \mathrm{C} 273^{\mathrm{b}}$, Ganymede $^{\mathrm{c}}$ \\
\hline
\end{tabular}

Notes.

a Array configuration: $\mathrm{C}=$ Compact; $\mathrm{E}=$ Extended; $\mathrm{V}=$ Very extended.

${ }^{\mathrm{b}}$ Calibrator: bandpass.

${ }^{\mathrm{c}}$ Calibrator: flux density scale.

previous $\mathrm{H} 92 \alpha$ and continuum measurements made with the VLA. Section 2 discusses the observations and data reduction. Section 3 presents our results and the modeling of the physical conditions and dynamics of the ionized gas in the vicinity of Sgr A*. Section 4 discusses the ionizing properties and possible links to the massive star formation in the central parsec, and Section 5 summarizes the results and conclusions. We assume a distance of $8 \mathrm{kpc}$ to $\mathrm{Sgr} \mathrm{A}^{*}$ throughout the paper. At this distance, 1 pc corresponds to $24^{\prime \prime}$.

\section{OBSERVATIONS AND DATA REDUCTION}

\subsection{SMA H30 $\alpha$ Line Data}

The $\mathrm{H} 30 \alpha$ line observations reported here were obtained with the SMA as part of a key project on the Galactic center in the period of 2006-2008. Our data comprise 11 tracks (Table 1). In all cases, the pointing center was the position of Sgr A*, and the half-power beam width at the $231.901 \mathrm{GHz}$ frequency of the $\mathrm{H} 30 \alpha$ recombination line was $53^{\prime \prime}$ ( 2 pc at the distance of Galactic center). The $2 \mathrm{GHz}$ bandwidth of the SMA correlator corresponds to a velocity range of $\pm 1300 \mathrm{~km} \mathrm{~s}^{-1}$. Six tracks were taken in the compact array configuration, where the synthesized beam was $5^{\prime \prime} .1 \times 33^{\prime \prime} 2$. Five higher-resolution tracks were also acquired, four in the extended configuration $\left(1^{\prime \prime} .5 \times 1\right.$ 1.1 resolution) and one in the very extended configuration $\left(0.7 \times 00^{\prime \prime} 4\right)$.

Calibrations of the data were carried out in Miriad (Sault et al. 1995) with the specific implementation for SMA data reduction. ${ }^{7}$ The system temperature correction was made in post-processing to compensate for amplitude attenuation owing to Earth's atmosphere. Using the calibrators listed in Table 1, we determined the antenna-based bandpass solutions for each observing track, and the bandpass corrections were applied to the visibility data. The flux density scale was determined from observations of planets. Phase corrections were made using the point source model of Sgr A*, conveniently located at the center of the field, based on self-calibrations. The continuum emission was subtracted from the spectral data using the Miriad task UVLIN for both the point source $(\sim 3 \mathrm{Jy})$ and the extended emission ( 3 Jy).

\footnotetext{
7 http://www.cfa.harvard.edu/sma/miriad
}

We constructed the $\mathrm{H} 30 \alpha$ line image cube with robust weighting $(R=2)$ (Briggs 1995). The synthesized FWHM beam was $1^{\prime \prime} .9 \times 1^{\prime \prime} .5\left(\right.$ P.A. $\left.=26^{\circ}\right)$. In order to compare the SMA images with the VLA images of the $\mathrm{H} 92 \alpha$ line and $22 \mathrm{GHz}$ continuum emission, the SMA image cube was convolved to a circular beam of $2^{\prime \prime}$. The intrinsic resolution of the correlator was $3.2 \mathrm{MHz}$, or $4.2 \mathrm{~km} \mathrm{~s}^{-1}$. We smoothed the spectra to a resolution of $15 \mathrm{~km} \mathrm{~s}^{-1}$ with a Miriad task. For this paper, we selected the velocity range of -360 to $+345 \mathrm{~km} \mathrm{~s}^{-1}$ for analysis. We found no significant emission beyond this range. The rms noise level of each channel image was $7 \mathrm{mJy}$. The image of the integrated $\mathrm{H} 30 \alpha$ line is shown in Figure 1 along with spectra in the directions of known infrared sources. Figure 2 shows the distribution of radial velocities determined from fitting the peak velocities of the $\mathrm{H} 30 \alpha$ line profile in the central $40^{\prime \prime}$ of the field. Notice the large velocity gradients along the Northern and Eastern Arms. The Western Arc does not show well in the SMA $\mathrm{H} 30 \alpha$ data because most of the line emission is below the SMA's $4 \sigma$ sensitivity limit. It is detected, however, in single-dish $\mathrm{H} 30 \alpha$ data taken with the IRAM 30 m telescope.

\subsection{VLA H92 $\alpha$ Line Data}

The detailed calibrations and imaging of the $\mathrm{H} 92 \alpha$ line data have been described by Zhao et al. (2009). They used the line image cube convolved to a circular beam $\left(\theta_{\mathrm{FWHM}}=2^{\prime \prime}\right)$, the same size as that of the $\mathrm{H} 30 \alpha$ line cube. Figure 3 shows a comparison of the spectra of the $\mathrm{H} 92 \alpha$ and $\mathrm{H} 30 \alpha$ lines toward selected regions containing IR sources. Table 2 lists the line fluxes and their ratios in these spectra.

\subsection{Continuum Data at $22 \mathrm{GHz}$}

The continuum image at $22 \mathrm{GHz}$ was obtained with the VLA high-resolution (A and B arrays) data set of 2005 described in Zhao et al. (2009) and the VLA archival data sets observed in $\mathrm{C}$ and D arrays during 2004 and 2005. The data reduction procedure described in Zhao et al. (2009) was followed. Sgr A* and the X-ray transient source (J174540.0290031) 3" south of Sgr A* were removed from the visibility data prior to imaging. The image of continuum emission was constructed from the visibility data with a long-baseline cutoff $(\leqslant 100 \mathrm{k} \lambda)$ to achieve a synthesized FWHM beam of $1 . .95 \times 1$ 1" 41 . The dirty image was 

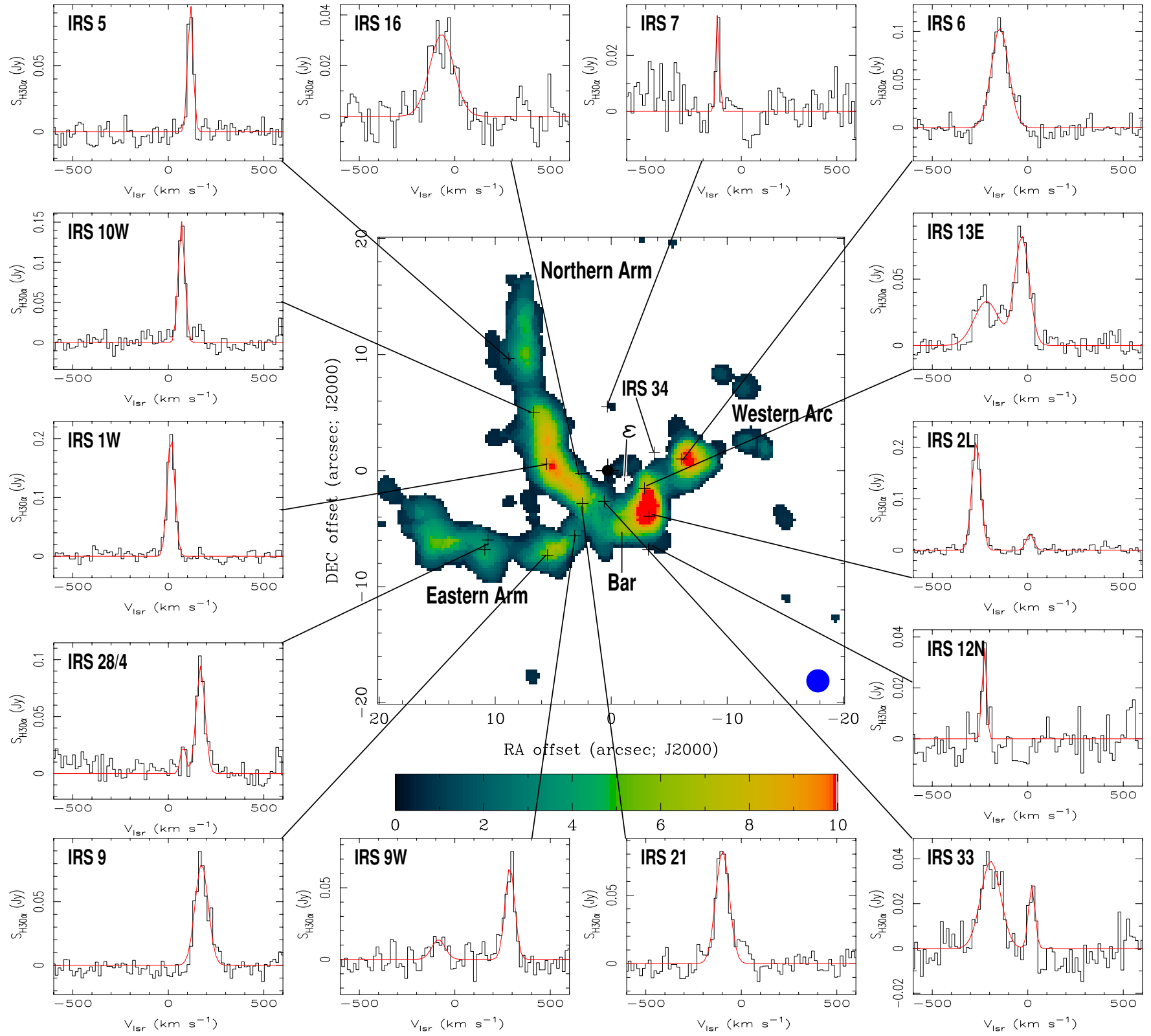

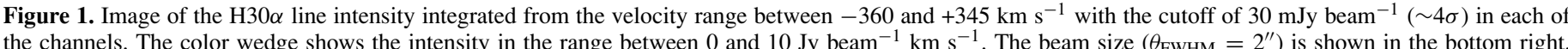

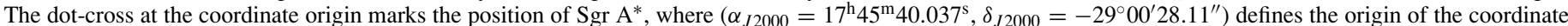

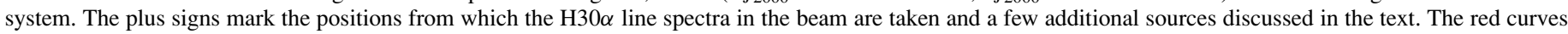

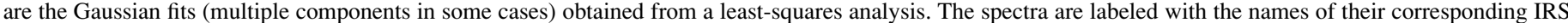
sources.

cleaned with the Clark-Steer hybrid algorithm and convolved to an FWHM beam of $2^{\prime \prime}$, for comparison with other data at the same resolution.

\subsection{IRAM H30 $\alpha$ Line Data}

We also used additional $\mathrm{H} 30 \alpha$ data taken with the HERA multibeam receiver on the IRAM $30 \mathrm{~m}$ telescope, with $11^{\prime \prime}$ beams, in order to calculate the global parameters for the region. Based on the intensity of integrated $\mathrm{H} 30 \alpha$ line emission in a lower-angular-resolution $\left(\sim 4^{\prime \prime}\right)$ image constructed by combining the SMA data with the IRAM $30 \mathrm{~m}$ data, we separated Sgr A West into two components: A, the bright minispiral features of the Northern and Eastern Arms $\left(\int S_{\mathrm{H} 30 \alpha} d V \geqslant 5 \mathrm{Jy}\right.$ beam ${ }^{-1} \mathrm{~km} \mathrm{~s}^{-1}$ ) that trace the high-density ionized gas, and $\mathrm{B}$, the residuals of Sgr A West after subtracting the emission from A. These results are in Table 2.

\section{RESULTS}

\subsection{Physical Conditions of the Ionized Gas in the Central Parsec}

The overall radio properties of the observed region (Sgr A West; $80^{\prime \prime} \times 45^{\prime \prime}$ ) determined from the $\mathrm{H} 30 \alpha, \mathrm{H} 92 \alpha$, and $22 \mathrm{GHz}$ continuum images are summarized in Table 2.

\subsubsection{Distribution of H3O $\alpha$ Line Emission and IR Sources}

Figures 1 and 2 show the $\mathrm{H} 30 \alpha$ line data in the central parsec. Figure 3 shows a comparison between the SMA H30 $\alpha$ 
Table 2

The H30 $\alpha$ \& H92 $\alpha$ Line Measurements

\begin{tabular}{|c|c|c|c|c|c|c|c|c|c|}
\hline \multirow[t]{2}{*}{ Region } & \multicolumn{9}{|c|}{ Radio Properties in IRS Regions } \\
\hline & $\begin{array}{c}\Delta \alpha \\
(\operatorname{arcsec})\end{array}$ & $\begin{array}{c}\Delta \delta \\
(\operatorname{arcsec})\end{array}$ & $\begin{array}{c}S_{\mathrm{H} 30 \alpha} \\
\left(\mathrm{Jy} \mathrm{beam}^{-1}\right)\end{array}$ & $\begin{array}{c}S_{22 \mathrm{GHz}} \\
\left(\mathrm{Jy} \mathrm{beam}^{-1}\right)\end{array}$ & $\frac{S_{\mathrm{H} 30 \alpha}}{S_{22 \mathrm{GHz}}}$ & $\frac{S_{\mathrm{H} 92 \alpha}}{S_{22 \mathrm{GHz}}}$ & $\frac{S_{\mathrm{H} 30 \alpha}}{S_{\mathrm{H} 92 \alpha}}$ & $\begin{array}{c}V_{\mathrm{LSR}^{\mathrm{a}}} \\
\left(\mathrm{km} \mathrm{s}^{-1}\right)\end{array}$ & $\begin{array}{l}\Delta V_{\mathrm{FWHM}^{\mathrm{a}}} \\
\left(\mathrm{km} \mathrm{s}^{-1}\right)\end{array}$ \\
\hline IRS $1 \mathrm{~W}$ & 5.27 & 0.57 & $0.21 \pm 0.008$ & $0.16 \pm 0.008$ & $1.3 \pm 0.08$ & $0.06 \pm 0.005$ & $21 \pm 1$ & $14 \pm 1$ & $46 \pm 2$ \\
\hline IRS 10W & 6.38 & 5.02 & $0.15 \pm 0.018$ & $0.10 \pm 0.003$ & $1.5 \pm 0.19$ & $0.07 \pm 0.004$ & $21 \pm 1$ & $69 \pm 2$ & $39 \pm 5$ \\
\hline IRS 5 & 8.53 & 9.63 & $0.10 \pm 0.019$ & $0.04 \pm 0.006$ & $2.5 \pm 0.6$ & $0.1 \pm 0.02$ & $25 \pm 2$ & $115 \pm 3$ & $33 \pm 8$ \\
\hline IRS 16 & 2.30 & -0.26 & $0.03 \pm 0.005$ & $0.08 \pm 0.015$ & $0.4 \pm 0.1$ & $0.026 \pm 0.008$ & $15 \pm 4$ & $-69 \pm 10$ & $141 \pm 27$ \\
\hline IRS 6 & -6.37 & 1.00 & $0.12 \pm 0.011$ & $0.15 \pm 0.006$ & $0.80 \pm 0.08$ & $0.034 \pm 0.003$ & $23 \pm 2$ & $-146 \pm 5$ & $109 \pm 13$ \\
\hline IRS13E & -3.17 & -1.53 & $0.08 \pm 0.006$ & $0.25 \pm 0.030$ & $0.32 \pm 0.05$ & $0.010 \pm 0.002$ & $32 \pm 4$ & $-31 \pm 3$ & $86 \pm 8$ \\
\hline IRS 2L & -3.57 & -3.93 & $0.21 \pm 0.011$ & $0.23 \pm 0.030$ & $0.91 \pm 0.13$ & $0.031 \pm 0.005$ & $29 \pm 1$ & $-269 \pm 1$ & $55 \pm 3$ \\
\hline IRS $12 \mathrm{~N}$ & -3.50 & -6.80 & $0.04 \pm 0.010$ & $0.04 \pm 0.002$ & $0.76 \pm 0.22$ & $0.036 \pm 0.013$ & $21 \pm 6$ & $-229 \pm 7$ & $40 \pm 17$ \\
\hline IRS 33 & 0.30 & -2.65 & $0.04 \pm 0.008$ & $0.19 \pm 0.024$ & $0.21 \pm 0.05$ & $0.009 \pm 0.003$ & $24 \pm 5$ & $-195 \pm 12$ & $126 \pm 29$ \\
\hline IRS 21 & 2.18 & -2.83 & $0.08 \pm 0.009$ & $0.16 \pm 0.018$ & $0.50 \pm 0.08$ & $0.023 \pm 0.005$ & $22 \pm 3$ & $-99 \pm 4$ & $80 \pm 11$ \\
\hline IRS 9W & 2.85 & -5.62 & $0.06 \pm 0.007$ & $0.11 \pm 0.011$ & $0.57 \pm 0.09$ & $\ldots \mathrm{b}$ & $\ldots \mathrm{b}$ & $288 \pm 4$ & $60 \pm 8$ \\
\hline IRS 9 & 5.20 & -7.30 & $0.08 \pm 0.009$ & $0.09 \pm 0.009$ & $0.85 \pm 0.13$ & $0.043 \pm 0.008$ & $20 \pm 2$ & $174 \pm 5$ & $84 \pm 11$ \\
\hline IRS 4 & 10.33 & -5.98 & $0.07 \pm 0.012$ & $0.05 \pm 0.006$ & $1.4 \pm 0.3$ & $0.07 \pm 0.02$ & $20 \pm 3$ & $164 \pm 4$ & $47 \pm 10$ \\
\hline IRS 28 & 10.60 & -6.80 & $0.10 \pm 0.010$ & $0.07 \pm 0.006$ & $1.5 \pm 0.2$ & $0.06 \pm 0.01$ & $25 \pm 2$ & $168 \pm 4$ & $49 \pm 22$ \\
\hline IRS 7 & 0.03 & 5.52 & $0.03 \pm 0.010$ & $0.15 \pm 0.001$ & $2.0 \pm 0.6$ & $0.07 \pm 0.03$ & $28 \pm 8$ & $-124 \pm 10$ & $29 \pm 22$ \\
\hline Minicavity $^{\mathrm{g}}$ & -1.75 & -2.80 & $0.08 \pm 0.006$ & $0.18 \pm 0.020$ & $0.45 \pm 0.07$ & $0.016 \pm 0.002$ & $29 \pm 2$ & $6 \pm 2$ & $41 \pm 4$ \\
\hline \multicolumn{10}{|c|}{ Radio Properties of Sgr A West } \\
\hline Component & \multicolumn{2}{|c|}{$\begin{array}{c}\int S_{\mathrm{H} 30 \alpha} d V \\
\left(\mathrm{Jy} \mathrm{km} \mathrm{s}^{-1}\right)\end{array}$} & \multicolumn{2}{|c|}{$\begin{array}{c}\int S_{\mathrm{H} 92 \alpha} d V \\
\left(\mathrm{Jy} \mathrm{km} \mathrm{s}^{-1}\right)\end{array}$} & \multicolumn{2}{|c|}{$\begin{array}{c}S_{22 \mathrm{GHz}} \\
(\mathrm{Jy})\end{array}$} & \multicolumn{2}{|c|}{ Area } & \\
\hline Sgr A West total ${ }^{\mathrm{c}}$ & \multicolumn{2}{|c|}{$1700 \pm 300^{\mathrm{d}}$} & \multicolumn{2}{|c|}{$60 \pm 7$} & \multicolumn{2}{|c|}{$16.3 \pm 0.2$} & $1.0 \pm 0.1$ & $5.5 \pm 0.5$ & \\
\hline $\mathrm{A}^{\mathrm{e}}$ & \multicolumn{2}{|c|}{$300 \pm 20$} & \multicolumn{2}{|c|}{$19 \pm 2$} & \multicolumn{2}{|c|}{$7.1 \pm 0.1$} & $0.10 \pm 0.01$ & $0.55 \pm 0.05$ & \\
\hline $\mathrm{B}^{\mathrm{f}}$ & \multicolumn{2}{|c|}{$1400 \pm 300$} & \multicolumn{2}{|c|}{$41 \pm 7$} & \multicolumn{2}{|c|}{$9.2 \pm 0.2$} & $1.0 \pm 0.1$ & $5.5 \pm 0.5$ & \\
\hline
\end{tabular}

Notes.

a Determined from the $\mathrm{H} 30 \alpha$ line.

b No H92 $\alpha$ data at the line velocity.

c The overall Sgr A West region $\left(80^{\prime \prime} \times 45^{\prime \prime}\right)$.

d The line flux determined from the IRAM 30 m observation.

e The bright $\mathrm{H} 30 \alpha$ line emission region (the Northern and Eastern Arms in Figure 2).

$\mathrm{f}$ The residual of Sgr A West after taking out the contribution from component A

$\mathrm{g}$ The line and continuum intensities listed in this row reflect averaged values of the emission from both the rim and the void of the minicavity in the $2^{\prime \prime}$ beam that is similar to the diameter of the minicavity.

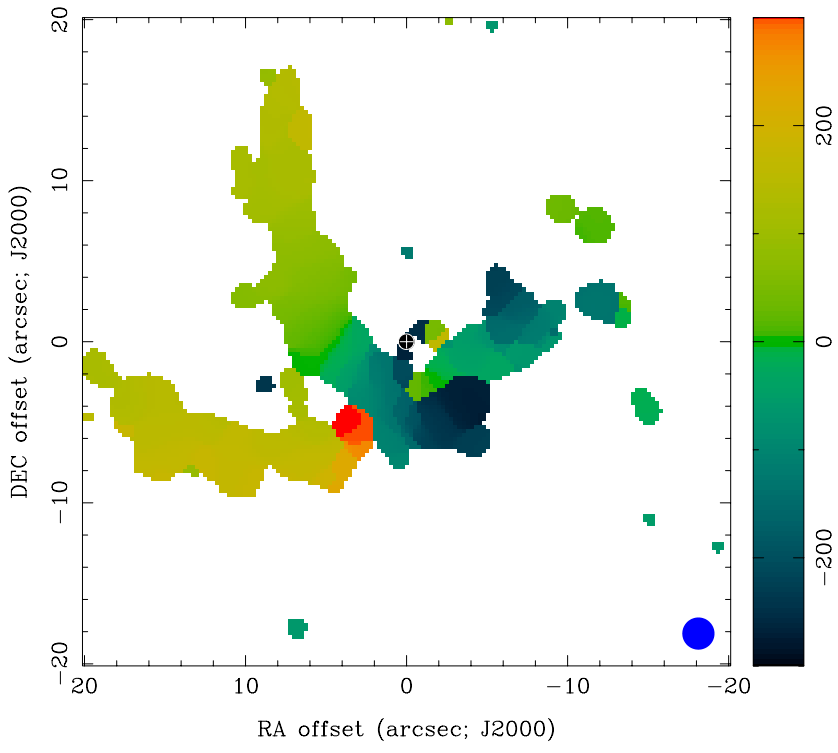

Figure 2. Distribution of the radial velocities determined from fitting the peak velocity of the $\mathrm{H} 30 \alpha$ line image cube observed with the SMA in the central $40^{\prime \prime}$ region. The color scale denotes velocity from -360 to $+345 \mathrm{~km} \mathrm{~s}^{-1}$. The circular beam $\theta_{\mathrm{FWHM}}=2^{\prime \prime}$ is shown at the bottom right. The dot-cross at the coordinate origin marks the position of $\mathrm{Sgr} \mathrm{A}^{*}$. and the VLA H92 $\alpha$ line spectra in the direction of selected IR sources. For optically thin gas in local thermodynamic equilibrium (LTE) with no pressure broadening, the ratio of line peak intensity is $R_{\mathrm{H} 92 \alpha}^{\mathrm{H} 30 \alpha} \equiv S_{\mathrm{H} 30 \alpha} / S_{\mathrm{H} 92 \alpha} \approx \nu_{\mathrm{H} 30 \alpha} / \nu_{\mathrm{H} 92 \alpha} \approx 28$. A detailed comparison of the $\mathrm{H} 30 \alpha$ line spectra from the SMA and the H92 $\alpha$ line spectra observed with the VLA yields line ratios in most of the regions that are close to the expected LTE ratio, suggesting that the ionized gas in the central parsec is optically thin and under LTE conditions. In some regions, however (e.g., IRS $1 \mathrm{~W}, 10 \mathrm{~W}, 16$, and 33), the observed ratios show significant departure from the expected LTE ratio.

\subsubsection{Distribution of $T_{e}^{*}$}

The equivalent electron temperature, $T_{e}^{*}$, derived from the ratio of line-to-continuum emission under the assumption that the optically thin gas is in LTE, can be estimated from our measurements. In order to avoid contamination from both the dust emission, which becomes dominant at higher frequencies, and significant synchrotron emission at lower frequencies, we used the continuum data at $22 \mathrm{GHz}$, where the contributions from both the synchrotron and thermal dust emission are negligible relative to the free-free emission. Figures 4(a) and (b) show the integrated $\mathrm{H} 30 \alpha$ line image and radio continuum image at $22 \mathrm{GHz}$, respectively. We assume that the continuum emission at $22 \mathrm{GHz}$ is entirely due to optically thin free-free 

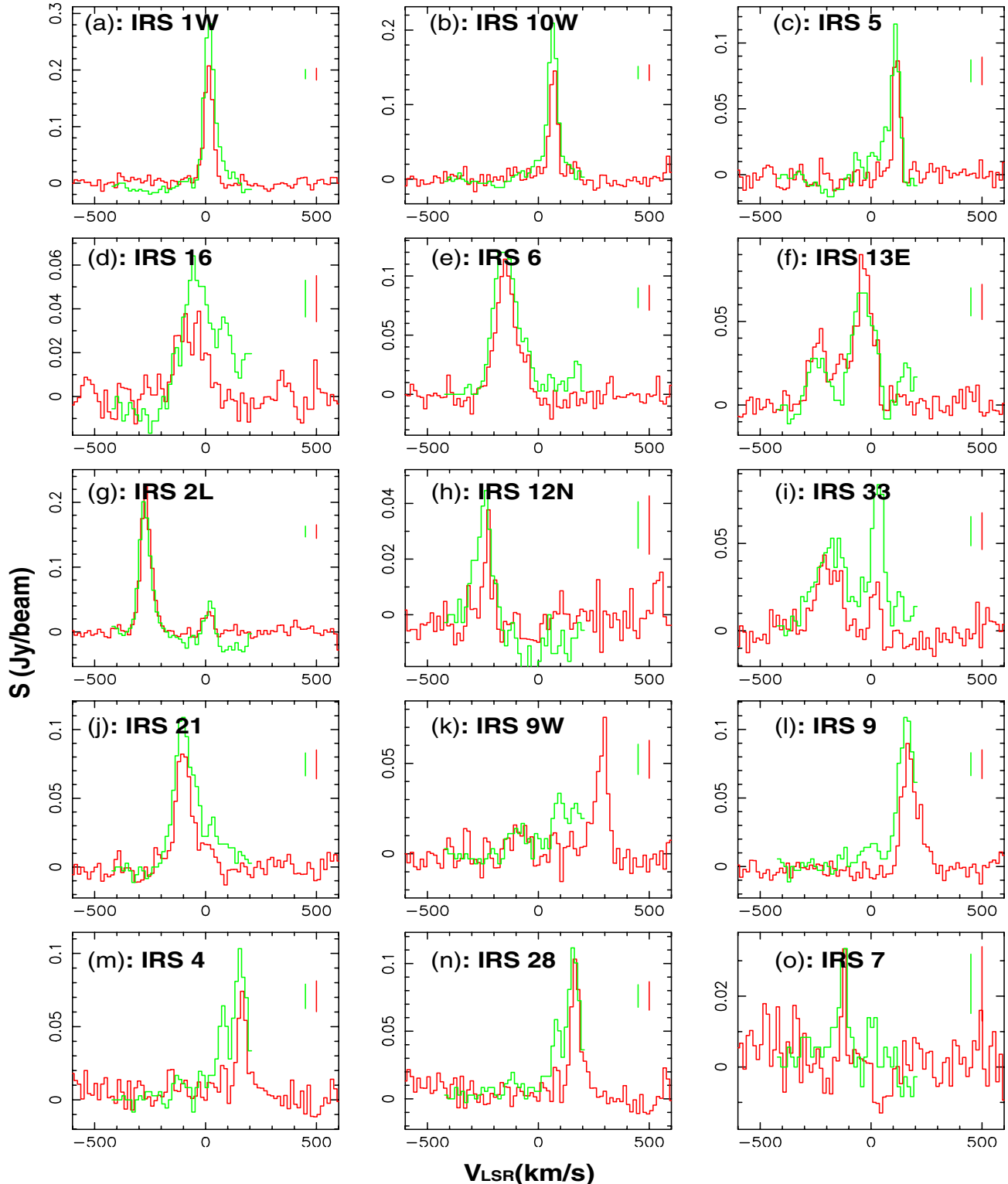

Figure 3. Comparison of the $\mathrm{H} 30 \alpha$ line spectra (red) with the VLA H92 $\alpha$ line spectra (green) taken from the selected IRS source regions with a resolution of $\theta_{\text {FWHM }}=2^{\prime \prime}$. The VLA H92 $\alpha$ spectra have been multiplied by a factor of $\nu_{\mathrm{H} 30 \alpha} / \nu_{\mathrm{H} 92 \alpha}=28$. The vertical bars indicate the uncertainty of $3 \sigma$.

(A color version of this figure is available in the online journal.)

emission and scale its intensity to $231.9 \mathrm{GHz}$, according to the power law $v^{-0.1}$, i.e., a factor of 0.79 . Using Equation (1) below (Wilson et al. 2009), we determined the value of $T_{e}^{*}$,

$$
\begin{aligned}
\frac{T_{e}^{*}}{K}= & {\left[\frac{6985}{a\left(v, T_{e}\right)}\left(\frac{v}{\mathrm{GHz}}\right)^{1.1} \frac{1}{1+N(\mathrm{He}) / N(\mathrm{H})}\right.} \\
& \left.\times\left(\frac{S_{C}}{S_{L} \Delta V_{\mathrm{FWHM}}}\right)\right]^{0.87},
\end{aligned}
$$

where $v$ is the frequency, $S_{L}$ is the line flux density, $S_{C}$ is the continuum flux density, $\Delta V_{\mathrm{FWHM}}$ is the line width (FWHM), and only Doppler broadening is involved, $N(\mathrm{He}) / N(\mathrm{H})$ is the number density ratio, which we take to be 0.08 , and $\alpha\left(v, T_{e}\right) \sim$ 0.97 (at $v=22 \mathrm{GHz}$ and $T_{e}$, the electron temperature, $\sim 10^{4} \mathrm{~K}$ ) is the correction to the Mezger \& Henderson (1967) powerlaw approximation. The angular distribution of $T_{e}^{*}$ is shown in Figure 4 (c). $T_{e}^{*}$ varies from $7000 \mathrm{~K}$ to $10,000 \mathrm{~K}$ in most regions of Sgr A West. However, in the Bar region (e.g., IRS 2L, IRS $12 \mathrm{~N}$, IRS 13E, and IRS 33), $3^{\prime \prime}$ southeast of Sgr A*, $T_{e}^{*}$ increases to $\sim 15,000 \mathrm{~K}$. The significant increase of $T_{e}^{*}$ in the Bar has also been derived from line observations of H92 $\alpha$ (Roberts \& Goss 1993) and H66 $\alpha$ (Schwarz et al. 1989).

\subsubsection{Isothermal Homogeneous H II Model}

Since the observed quantities of line and continuum intensities depend on the density, temperature, and path length of H II gas, we can construct a model and fit the data to it over a wide frequency range in order to determine the parameters of the ionized gas. In particular, with the high-resolution data obtained from interferometer observations, uncertainties due to variations in structure and the physical parameters across the source are substantially reduced. Thus, the results from such an analysis become more reliable. Models for RRL emission from the nuclear region of external galaxies have been discussed by Puxley et al. (1991), Anantharamaiah et al. (1993), 

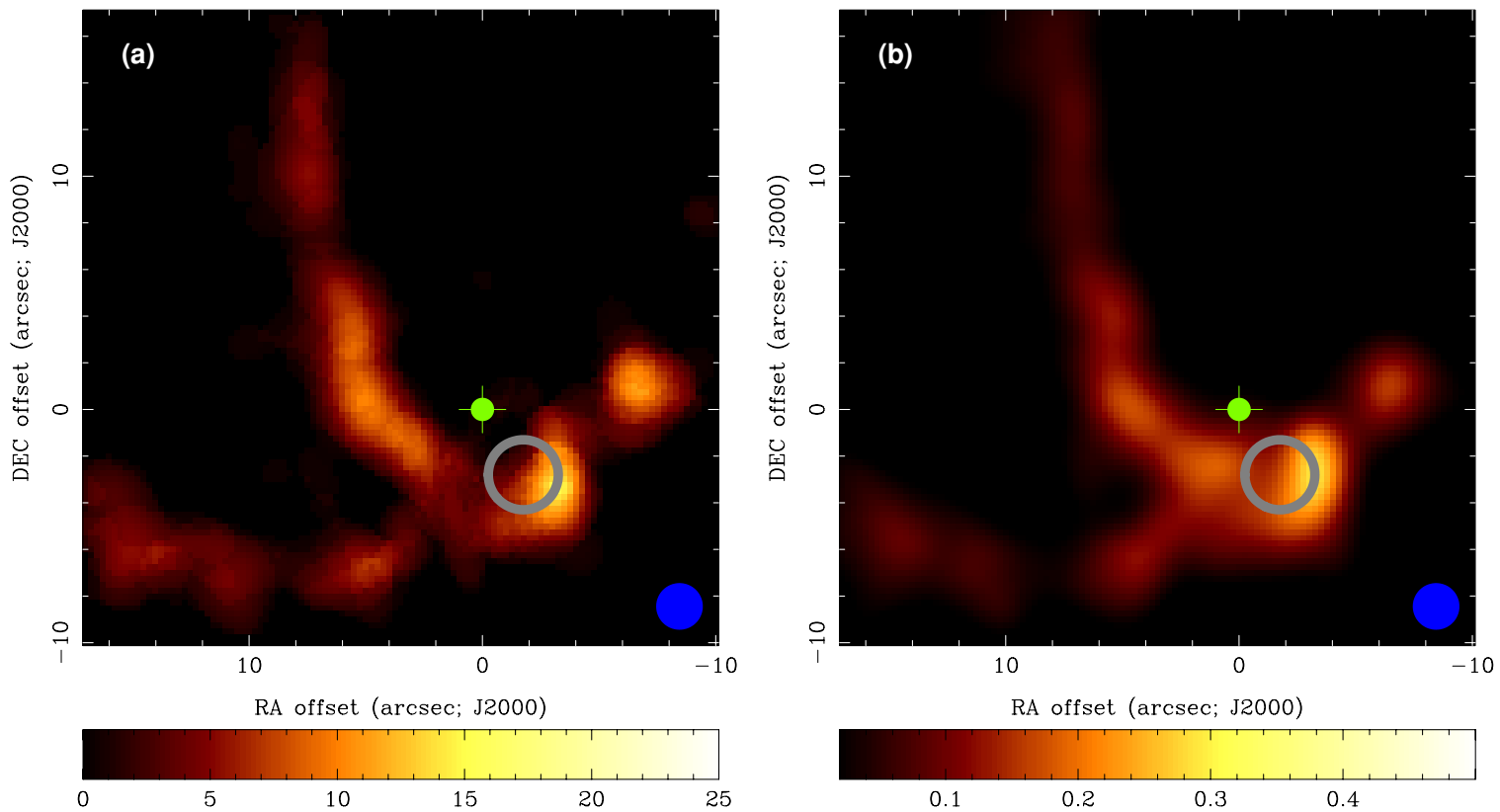

RA offset (arcsec; J2000)
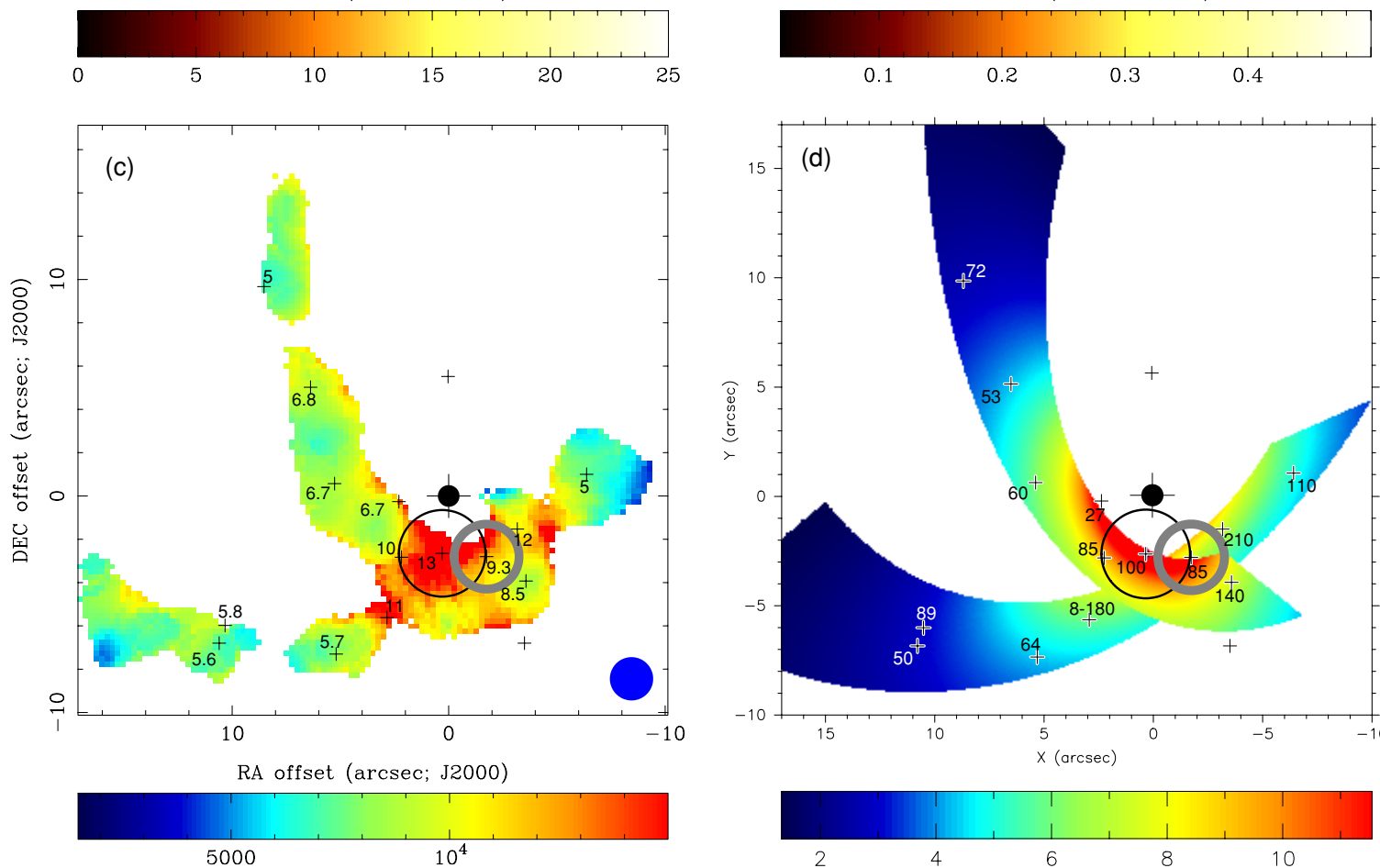

Figure 4. (a) Integrated $\mathrm{H} 30 \alpha$ line emission observed at $1.3 \mathrm{~mm}$ with the SMA. (b) Radio continuum image made from VLA observations at $1.3 \mathrm{~cm}$ with a resolution of $\theta_{\mathrm{FWHM}}=2^{\prime \prime}$. The radio emission associated with Sgr A* and the X-ray transient (J174540.0290031) has been removed, but the position of Sgr A* is marked by the dot-cross at the coordinate origin in each of the figures. (c) Pseudo-color shows the LTE temperature $\left(T_{e}^{*}\right)$ derived from images of H30 $\alpha$ line emission and radio continuum based on Equation (1). (d) The image of the specific kinetic energy computed using the Keplerian orbital model described in Table 5 of Zhao et al. (2009). The color scale shows the specific kinetic energy in units of $10^{14} \mathrm{erg} \mathrm{g}^{-1}$. In Figures 3(c) and (d), the electron temperature $\left(T_{e}\right.$ in $\left.10^{3} \mathrm{~K}\right)$ and the electron density $\left(n_{e}\right.$ in $10^{3} \mathrm{~cm}^{-3}$ ) are shown at the positions of selected IR sources. These numbers are derived using the isothermal homogeneous $\mathrm{H}$ II model with the non-LTE effects. The results are tabulated in Table 3. The thick gray circles in (a)-(d) indicate the well-known minicavity region (Yusef-Zadeh et al. 1989); the black circles in (c) and (d) indicate high temperature regions.

(A color version of this figure is available in the online journal.)

Anantharamaiah et al. (2000), Zhao et al. (1996), Zhao et al. (1997), Phookun et al. (1998), and Rodriguez-Rico et al. (2004). They found that the main constraints for the models are the integrated RRL strength at multiple frequencies and the observed radio continuum spectrum. The observed quantities (line and continuum intensities) depend in a nonlinear way on the distribution of $T_{e}$ and $n_{e}$, the electron density, both along the line of sight and across the telescope beam. In the cases of the extragalactic nuclear regions, at a given frequency, the line emission often arises from $\mathrm{H}$ II components under the physical conditions that are particularly favorable at this particular frequency. Usually, multiple collections of $\mathrm{H}$ II regions with different physical parameters are required to fit the observations.

Both the SMA and VLA data achieve angular resolution ( $2^{\prime \prime}$, $\sim 0.1 \mathrm{pc}$ ) adequate to resolve the $\sim 1 \mathrm{pc}$ minispiral structure. The effects due to changes of physical parameters across the source are therefore less significant. However, the electron temperature and density could possibly change along the line of sight; $T_{e}$ and $n_{e}$ derived from the simple isothermal and homogeneous model correspond, therefore, to average physical properties of the $\mathrm{H}$ II 
gas in the telescope beam. If the $\mathrm{H}$ II gas in the beam along the path length is isothermal and homogeneous, then the line and continuum flux densities from a region subtending a solid angle $\Omega$ are given by (e.g., Shaver 1975)

$$
S_{L}=\frac{2 k v^{2}}{c^{2}} \Omega T_{e}\left[\left(\frac{\tau_{L} / \beta_{n}+\tau_{C}}{\tau_{L}+\tau_{C}}\right)\left(1-e^{-\left(\tau_{L}+\tau_{C}\right)}\right)-\left(1-e^{-\tau_{C}}\right)\right],
$$

and

$$
S_{C}=\frac{2 k v^{2}}{c^{2}} \Omega T_{e}\left(1-e^{-\tau_{C}}\right),
$$

where $k$ is Boltzmann's constant, $c$ is the speed of light, and the line $\left(\tau_{L}\right)$ and continuum $\left(\tau_{C}\right)$ optical depths are given by

$$
\begin{aligned}
\tau_{L} \approx & 575 b_{n} \beta_{n}\left(\frac{v}{\mathrm{GHz}}\right)^{-1}\left(\frac{n_{e}}{\mathrm{~cm}^{-3}}\right)^{2} \\
& \times\left(\frac{L f_{V}}{\mathrm{pc}}\right)\left(\frac{T_{e}}{\mathrm{~K}}\right)^{-5 / 2}\left(\frac{\Delta V_{D}}{\mathrm{~km} \mathrm{~s}^{-1}}\right)^{-1}\left(1+1.48 \frac{\Delta V_{P}}{\Delta V_{D}}\right)^{-1},
\end{aligned}
$$

and

$$
\begin{aligned}
\tau_{C} \approx & 0.08235\left(\frac{n_{e}}{\mathrm{~cm}^{-3}}\right)^{2}\left(\frac{L f_{V}}{\mathrm{pc}}\right)\left(\frac{v}{\mathrm{GHz}}\right)^{-2.1} \\
& \times\left(\frac{T_{e}}{\mathrm{~K}}\right)^{-1.35} a\left(v, T_{e}\right)
\end{aligned}
$$

respectively. $b_{n}$ and $\beta_{n}$ are the population departure coefficients, $n_{e}$ is the electron density, $\Delta V_{D}$ and $\Delta V_{P}$ are the FWHM Doppler and pressure line widths in $\mathrm{km} \mathrm{s}^{-1}$, respectively, $L$ is the path length, and $f_{V}$ is the volume filling factor. Note that the last factor in Equation (4) provides a convenient approximation for the transition between the pure Doppler and pure pressurebroadened cases.

The Doppler broadening has a thermal and turbulent component and is given by

$$
\Delta V_{D}=\sqrt{\frac{8 \ln (2) k T_{e}}{M_{\mathrm{H}}}+\left(\Delta V_{t}\right)^{2}},
$$

where $M_{\mathrm{H}}$ is the mass of hydrogen and $\Delta V_{t}$ is the turbulent width, which here includes microscopic turbulence and dynamical velocity gradients. In the central parsec, the thermal width $\left(\Delta V_{\text {th }}=\sqrt{\frac{8 \ln (2) k T_{e}}{M_{\mathrm{H}}}}\right)$ is less than the turbulent width $\left(\Delta V_{t}\right)$. The line width due to pressure broadening, computed by Brocklehurst \& Leeman (1971), is given by

$$
\Delta V_{P}=1.7 \times 10^{-18} \frac{n_{e}}{T_{e}^{0.1}} N^{7.4} \mathrm{~km} \mathrm{~s}^{-1},
$$

where $N$ is the principal quantum number of the transition. The total line width is approximately the quadratic sum of $\Delta V_{D}$ and $\Delta V_{P}$,

$$
\Delta V_{\mathrm{FWHM}} \cong \sqrt{\left(\Delta V_{D}\right)^{2}+\left(\Delta V_{P}\right)^{2}} .
$$

$\Delta V_{\mathrm{FWHM}}$ is an observable quantity. We solved for the parameters $T_{e}, n_{e}, \Delta V_{t}$, and $L f_{V}$ for each point in the image in the following way. Since $\Delta V_{P} \ll \Delta V_{\text {th }}<\Delta V_{t}$ for reasonable values of $T_{e}$ and $n_{e}$, we assume initial values of $T_{e}$ and $n_{e}$, and solve for $\Delta V_{t}$ via Equations (6)-(8). We computed the values for $b_{n}$ and $\beta_{n}$ for the assumed values of $T_{e}$ and $n_{e}$ using the NLTE code of Gordon \& Sorochenko (2002), which followed the analysis of Walmsley (1990). We then formed the flux density ratios $R_{22 \mathrm{GHz}}^{\mathrm{H} 30 \alpha} \equiv S_{\mathrm{H} 30 \alpha} / S_{22 \mathrm{GHz}}, R_{22 \mathrm{GHz}}^{\mathrm{H} 92 \alpha} \equiv S_{\mathrm{H} 92 \alpha} / S_{22 \mathrm{GHz}}$, and $R_{92 \alpha}^{\mathrm{H} 30 \alpha} \equiv S_{\mathrm{H} 30 \alpha} / S_{\mathrm{H} 92 \alpha}$. The use of these ratios eliminates the solid angle $\Omega$ in Equations (2) and (3). At each point in the image, we used these ratios to solve for $T_{e}, n_{e}$, and $L f_{V}$. We then iterated the process, i.e., solved for a new estimate of $\Delta V_{t}$ with these values of $T_{e}$ and $n_{e}$. The process converged in a few iterations.

The parameters derived for the line of sight for each of the regions are summarized in Table 3. Instead of $L f_{V}$, we report the $\mathrm{EM}\left(\mathrm{EM}=n_{e}^{2} L f_{V}\right)$. The ratio $T_{e} / T_{e}^{*}$ is given in Column 6 , which mainly corrects for the effect due to underpopulated electrons at the level $N=30$ as compared to that expected under the LTE; the ratio $\Delta V_{t} / \Delta V_{\mathrm{FWHM}}$ in Column 7 ; and $\Delta V_{P}^{\mathrm{H} 92 \alpha} / \Delta V_{D}^{\mathrm{TH}}$, the ratio of the $\mathrm{H} 92 \alpha$ line width of pressure broadening to that of thermal Doppler broadening, in Column 8 . The pressure broadening term $\left(\Delta V_{P}^{\mathrm{H} 30 \alpha}\right)$ is negligible.

The uncertainties in the physical parameters mainly come from the uncertainties in the measurements. Although the relation between the physical parameters and observable quantities is nonlinear, we assess the errors through linear approximation. We use the optically thin assumption, which appears to be valid at the frequencies used in this paper. The fractional uncertainty in $T_{e}$, which is proportional to the fractional uncertainties of the measured ratios, $R_{22 \mathrm{GHz}}^{\mathrm{H} 30 \alpha}$ and $R_{\mathrm{H} 92 \alpha}^{\mathrm{H} 30 \alpha}$, lies in the range from $7 \%$ for IRS $1 \mathrm{~W}$ to $39 \%$ for IRS $12 \mathrm{~N}$. The fractional uncertainty in $n_{e}$ is dominated by those of observed line ratios $R_{\mathrm{H} 92 \alpha}^{\mathrm{H} 30 \alpha}$ and ranges from $6 \%$ for IRS $1 \mathrm{~W}$ to $36 \%$ for IRS $12 \mathrm{~N}$.

The variations of the $\mathrm{H} 30 \alpha$ line intensities reflect the changes of excitation conditions in the region. Our model fitting suggests that, given similar solutions in $n_{e}$ and $T_{e}$, the large difference in the $\mathrm{H} 30 \alpha$ line intensity between the two extreme regions (IRS $1 \mathrm{~W}$ and IRS 7) is mainly due to the difference of the EM or the path length along the line of sight (see Table 3).

In short, the line ratio of $\mathrm{H} 30 \alpha$ to $\mathrm{H} 92 \alpha$ varies from $15 \pm 4$ to $32 \pm 4$. The derived mean values of the line optical depth are $\bar{\tau}_{L}(\mathrm{H} 30 \alpha)=-0.001$ and $\bar{\tau}_{L}(\mathrm{H} 92 \alpha)=-0.006$ with standard deviations of $\sigma_{\tau_{L}(\mathrm{H} 30 \alpha)}=0.0004$ and $\sigma_{\tau_{L}(\mathrm{H} 92 \alpha)}=0.005$ from the corresponding emission regions. The free-free optical depth at the $\mathrm{H} 30 \alpha$ line frequency $\tau_{C}(231.9 \mathrm{GHz})$ varies from $1 \times 10^{-4}$ to $1 \times 10^{-5}$ while $\tau_{C}(8.3 \mathrm{GHz})$ is in the range between 0.16 and 0.01 . Because of the small optical depths in both line and continuum, the non-LTE effects in the minispiral, in general, are not critical.

There is a diffuse nonthermal synchrotron component in the central parsec region (Ekers et al. 1983). Based on the flux density of $22 \mathrm{Jy}$ at $20 \mathrm{~cm}$ in the central $40^{\prime \prime} \times 70^{\prime \prime}$ from Ekers et al. (1983), we estimated that the contribution to the continuum flux density in a $2^{\prime \prime}$ beam is $\sim 10 \mathrm{mJy}$ under the assumption that the nonthermal spectral index $\alpha \sim-0.5\left(S_{v} \propto v^{\alpha}\right)$ and the emission are evenly distributed. The optical depth of the $\mathrm{H} 92 \alpha$ line is small $\left(\tau_{L} \sim-0.01\right)$. The contribution from the stimulated H92 $\alpha$ emission of $\sim 0.1 \mathrm{mJy}$ is insignificant in the regions discussed in this paper. The contribution from the stimulated line emission by the dust emission at $232 \mathrm{GHz}$ is negligible because of the small optical depth of the $\mathrm{H} 30 \alpha$ line.

The observed line widths of the $\mathrm{H}$ II gas in the minispiral arms at the Galactic center are dominated by $\Delta V_{t}$, which is consistent with velocity gradients due to gas motion around $\mathrm{Sgr} \mathrm{A}^{*}$ or the SMBH being primarily responsible for the broadening in the line profiles observed in the $2^{\prime \prime}$ beam. 
Table 3

Physical Conditions of Ionized Gas in IRS Regions

\begin{tabular}{|c|c|c|c|c|c|c|c|}
\hline Region & $\begin{array}{c}T_{\mathrm{e}} \\
\left(10^{3} \mathrm{~K}\right)\end{array}$ & $\begin{array}{c}n_{\mathrm{e}} \\
\left(10^{4} \mathrm{~cm}^{-3}\right)\end{array}$ & $\begin{array}{c}\text { EM } \\
\left(10^{7} \mathrm{~cm}^{-6} \mathrm{pc}\right)\end{array}$ & $\begin{array}{c}\tau_{\mathrm{C}}(22 \mathrm{GHz}) \\
\left(10^{-3}\right)\end{array}$ & $\left(\frac{T_{\mathrm{e}}}{T_{\mathrm{e}}^{*}}\right)^{\mathrm{a}}$ & $\left(\frac{\Delta V_{t}}{\Delta V_{\mathrm{FWHM}}}\right)$ & $\left(\frac{\Delta V_{\mathrm{P}}^{\mathrm{H} 92 \alpha}}{\Delta V_{\mathrm{D}}^{\mathrm{TH}}}\right)^{\mathrm{b}}$ \\
\hline IRS $1 \mathrm{~W}$ & $6.7 \pm 0.5$ & $6.0 \pm 0.4$ & $1.8 \pm 0.1$ & 14. \pm 1.1 & 0.71 & 0.88 & 0.82 \\
\hline IRS $10 \mathrm{~W}$ & $6.8 \pm 0.6$ & $5.3 \pm 0.4$ & $1.1 \pm 0.1$ & $8.5 \pm 1.0$ & 0.71 & 0.85 & 0.72 \\
\hline IRS 5 & $5.0 \pm 1.1$ & $7.2 \pm 1.2$ & $0.4 \pm 0.1$ & $4.7 \pm 1.3$ & 0.68 & 0.78 & 1.2 \\
\hline IRS 16 & $6.7 \pm 2.0$ & $2.7 \pm 0.7$ & $0.8 \pm 0.2$ & $6.4 \pm 2.7$ & 0.68 & 0.99 & 0.37 \\
\hline IRS 6 & $5.0 \pm 0.6$ & 11. \pm 1.1 & $1.6 \pm 0.1$ & 18. \pm 2.3 & 0.71 & 0.96 & 1.8 \\
\hline IRS 13E & 12. \pm 2.5 & 21. \pm 3.6 & $3.2 \pm 0.5$ & 12. \pm 1.9 & 0.84 & 0.84 & 2.0 \\
\hline IRS 2L & $8.5 \pm 1.3$ & 14. \pm 1.4 & $2.7 \pm 0.4$ & 16. \pm 2.9 & 0.78 & 0.80 & 1.7 \\
\hline IRS $12 \mathrm{~N}$ & 12. \pm 4.7 & $2.8 \pm 1.0$ & $0.5 \pm 0.2$ & $1.8 \pm 0.6$ & 0.77 & 0.80 & 0.27 \\
\hline IRS 33 & 13. \pm 3.3 & 10. \pm 3.0 & $2.6 \pm 0.3$ & $8.5 \pm 1.8$ & 0.81 & 0.97 & 0.92 \\
\hline IRS 21 & 10. \pm 1.8 & $8.5 \pm 1.3$ & $2.0 \pm 0.3$ & $9.1 \pm 2.0$ & 0.78 & 0.94 & 0.92 \\
\hline IRS 9W & 11. -12 . & $0.8-18$ & $1.3-1.3$ & $5.5-4.9$ & $0.74-0.83$ & $0.76-0.93$ & $1.7-0.08$ \\
\hline IRS 9 & $5.7 \pm 0.9$ & $6.4 \pm 0.8$ & $1.0 \pm 0.2$ & $9.7 \pm 1.8$ & 0.69 & 0.97 & 0.97 \\
\hline IRS 4 & $5.8 \pm 1.3$ & $5.0 \pm 1.0$ & $0.5 \pm 0.1$ & $4.8 \pm 1.4$ & 0.68 & 0.91 & 0.75 \\
\hline IRS 28 & $5.6 \pm 0.8$ & $8.9 \pm 1.0$ & $0.7 \pm 0.1$ & $6.8 \pm 1.1$ & 0.71 & 0.86 & 1.4 \\
\hline IRS 7 & $7.0 \pm 2.5$ & $8.8 \pm 2.7$ & $0.2 \pm 0.1$ & $1.2 \pm 0.5$ & 0.74 & 0.64 & 1.2 \\
\hline Minicavity & $9.3 \pm 0.9$ & $8.5 \pm 0.7$ & $2.0 \pm 0.3$ & 10. \pm 2.0 & 0.77 & 0.78 & 0.96 \\
\hline
\end{tabular}

Notes. ${ }^{\text {a }} \frac{T_{\mathrm{e}}}{T_{\mathrm{e}}^{*}} \approx\left[b_{30}\left(1-\frac{1}{2} \beta_{30} \tau_{\mathrm{C}}(232 \mathrm{GHz})\right]^{0.87} \approx b_{30}^{0.87}\right.$ for a very small value of $\tau_{\mathrm{C}}(232 \mathrm{GHz})$ in a region at the Galactic center. ${ }^{\mathrm{b}} \Delta V_{\mathrm{D}}^{\mathrm{TH}}=\sqrt{\frac{8 \ln (2) k T_{e}}{M_{H}}}$ is the thermal Doppler line width.

\subsubsection{Northern and Eastern Arms}

The ionized gas of the high-density components in the Northern and Eastern Arms has a mean kinetic temperature of $\bar{T}_{e} \approx 6 \times 10^{3} \mathrm{~K}$ and an electron density of $n_{e}$ in the range of $10^{4-5} \mathrm{~cm}^{-3}$, which is consistent with the values $\left(10^{4} \mathrm{~cm}^{-3}\right)$ derived from $\mathrm{Pa} \alpha$ line emission at $1.87 \mu \mathrm{m}$ (Scoville et al. 2003) for a given uncertainty in extinction corrections in the near-IR. The H II gas properties in the Northern and Eastern Arms are similar to these in $\mathrm{H}$ II regions around young and/or evolved massive stars.

\subsubsection{The Bar}

We confirm that the ionized gas in the Bar (e.g., IRS 2L, IRS $12 \mathrm{~N}$, IRS 13E, and IRS 33 in Table 3) appears to have a higher kinetic temperature in the range of $8.5 \times 10^{3} \mathrm{~K}$ $\leqslant T_{e} \leqslant 1.3 \times 10^{4} \mathrm{~K}$, as suggested by earlier RRL observations (Roberts \& Goss 1993). The electron density shows a range from a few times $10^{4}$ to a few times $10^{5} \mathrm{~cm}^{-3}$.

The heating process may be complicated in the Bar because of complex gas interactions. There are several energy sources in the region. First, strong winds from the central stellar clusters such as IRS 16 and IRS 13 could provide kinetic energy to compress the ionized gas in this region. The heating due to the interaction between the winds and the ionized gas in the Bar likely occurs in the regions localized to the cluster sources.

In addition, the well-known minicavity (marked with the gray circle in Figures 4(a) and (b)), a region depressed of radio continuum, is located 3."5 southwest of Sgr A* (Yusef-Zadeh et al. 1989). This region is likely created by a fast wind outflow from the sources in the vicinity sweeping up the gas in the orbiting ionized streams, as suggested by the morphology of a bright [Fe III] line in the infrared (Lutz et al. 1993). However, the minicavity is a few arcsec displaced from the high-kinetictemperature region, which in fact is best seen as a depression associated with $\mathrm{H} 30 \alpha$ line emission. The minicavity can also be explained by Melia et al. (1996) as a downstream, focused flow due to the accretion by the SMBH at Sgr A*.
Alternatively, the high temperature in the Bar could result from a collision between two ionized orbiting flows, as suggested in the dynamical model of Zhao et al. (2009). A computation using their Keplerian orbital model (see Section 3.2) shows that the specific kinetic energy reaches a maximum (see Figure 4(d)) in the Bar region where high kinetic temperatures (see Figure 4(c)) are observed. The distribution of specific kinetic energy (Figure 4(d)) shows a substantial increase from $2 \times 10^{14} \mathrm{erg} \mathrm{g}^{-1}$ at a region near IRS 5 in the Northern Arm to $12 \times 10^{14} \mathrm{erg} \mathrm{g}^{-1}$ at IRS 33 in the Bar. However, we note that a high kinetic energy of the orbiting flow does not have to correspond to a high kinetic temperature; the ionized flows can release their kinetic energy to shocks if the ionized flows collide with each other. If they collide with the strong stellar winds from the clusters of stars in the Bar area, then the gas could be heated by the shocks to higher temperatures. In addition, the model also shows that the ionized flows of the Northern and Eastern Arms do collide at the region with high kinetic temperatures (see Figure 21(d) of Zhao et al. (2009) and the three-dimensional structure of the minispiral arms in Figure 5). The observed high kinetic temperatures in the Bar suggest that the ionized gas could be further heated by the shocks generated from the collision.

\subsection{Kinematics and Dynamics}

Kinematics of the ionized gas in both the Northern Arm and Western Arc have been explained as Keplerian circular motion of a circumnuclear ring (Schwarz et al. 1989; Lacy et al. 1991; Vollmer \& Duschl 2000; Liszt 2003). Based on the VLA observations of H92 $\alpha$, Roberts \& Goss (1993) found that the ionized ring consists of three separated kinematic components, the Western Arc, Northern Arm, and Bar. The radial velocities of the Western Arc can be fitted with a Keplerian model with circular motion. Using the observations of the Brackett- $\gamma$ line, Paumard et al. (2004) found that the kinematics of the ionized gas in the Northern Arm can be explained as a bundle of elliptical orbits. In addition, the Eastern Arm may be interrupted by a high-velocity component "tip." Also, the Bar component 


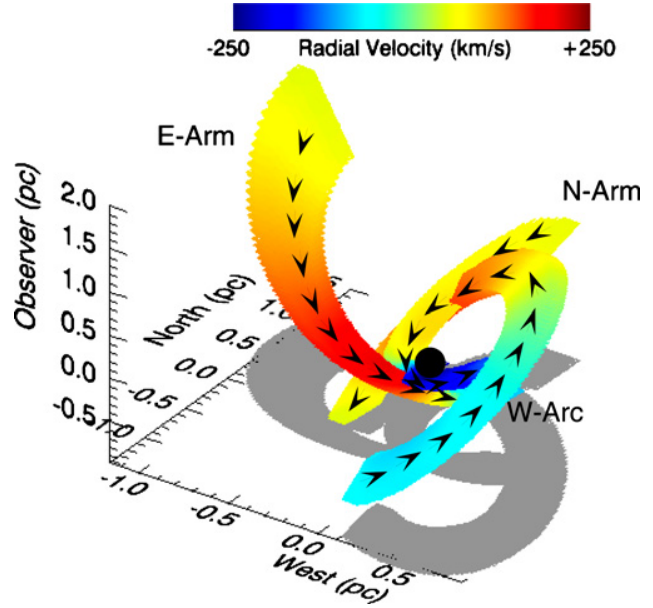

Figure 5. Three-dimensional view of the minispiral structure and velocity along each of the minispiral arms in the nucleus of the Galaxy centered at Sgr A* (black dot), which are computed using the Keplerian model described in Zhao et al. (2009). The orbital parameters were derived from fitting the VLA H92 $\alpha$ line data and are summarized in Table 5 of Zhao et al. (2009). The arrows indicate the direction of the ionized flows along the orbits. The color illustrates the radial velocity between -250 and $+250 \mathrm{~km} \mathrm{~s}^{-1}$ (color wedge at top). The gray shadow indicates the loci of the ionized flows projected on the sky plane.

(A color version of this figure is available in the online journal.)

extends to the IRS $6 / 34$ region, which is affected by two other kinematic features, the "Western Bridge" and the "Bar Overlay." The VLA observations of the $\mathrm{H} 92 \alpha$ line suggest that the loci of the three minispiral arms can be fitted with three families of elliptical orbits; the orbits of both the Northern and Eastern Arms have high eccentricities while the Western Arc is in nearly circular motion (Zhao et al. 2009). The VLA measurements of the proper motions and the radial velocity resolved the degeneracies in the inclination angles, suggesting that the two ionized flows from the Northern and Eastern Arms collide in the Bar region.

On the other hand, Mužić et al. (2007) suggested that the nonKeplerian motions might be important in the kinematics of the filaments observed near Sgr A*. In addition, Vollmer \& Duschl (2000) noticed that a substantial amount of velocity dispersion along with additional inward radial motion is required in order to fit the observed velocities of the ionized flows with their Keplerian circular-orbit model.

\subsubsection{Three-dimensional View of the Minispiral Structure and the Circumnuclear Disk}

Using the Keplerian model described in Zhao et al. (2009) along with the orbital parameters derived from fitting the VLA H92 $\alpha$ data and proper motion measurements, we recomputed the three-dimensional locations of the three ionized arms. Figure 5 shows a three-dimensional visualization of the three ionized flows in the Northern, Eastern, and Western Arms (Arc), which illustrates that the Northern Arm and Western Arc are nearly coplanar based on the similar longitudes of their ascending nodes $(\Omega)$ and inclination angles $(i)$ derived from fitting the loci of the ionized flows. The mean values of the two orbital angles, $\bar{\Omega}=71^{\circ} \pm 6^{\circ}$ and $\bar{i}=119^{\circ} \pm 3^{\circ}$, are inferred for the two coplanar orbits (Northern Arm and Western Arc), which form the ionized ring. The orbital plane of the ionized flows appears to be aligned with the plane of the circumnuclear disk (CND) derived by Güsten et al. (1987) from molecular line observations. If the fitted parameters from both the molecular lines (Güsten et al. 1987) and hydrogen recombination lines
(Schwarz et al. 1989; Lacy et al. 1991; Roberts \& Goss 1993; Vollmer \& Duschl 2000; Liszt 2003; Zhao et al. 2009) are correct, the Keplerian motions dominate the kinematics in the central parsec, and the plane of the main nuclear gaseous disk or the CND can be described by the mean orbital angles inferred above. Therefore, the orbital plane of the Eastern Arm ( $i=122^{\circ} \pm 5^{\circ}$ and $\Omega=-42^{\circ} \pm 11^{\circ}$ ) is nearly perpendicular to that of the CND. Figure 5 shows that the Eastern Arm flow runs into the CND from the northeast and the near side with respect to Sgr A* and collides with the Northern Arm flow in the region located south and behind Sgr A*.

\subsubsection{Kinematics and Keplerian Motions}

Figure 6 shows the spectra taken from 12 positions along each of the two ionized arms (shown in Figure 7). The observed spectra are compared with the radial velocities (thick green bars) derived from the Keplerian model. Note that we assume that the LSR velocity of the black hole, $V_{z}$, is 0 to within $\pm 5 \mathrm{~km} \mathrm{~s}^{-1}$ (see discussion in Gillessen et al. 2009). In general, the kinematics of the ionized gas follow the Keplerian orbital motion expected for the gravity of the SMBH $\left(4.2 \times 10^{6} M_{\odot}\right)$ at $\mathrm{Sgr} \mathrm{A}^{*}$. Models of the enclosed mass (see Oh et al. 2009) show that the added enclosed mass starts to rise above the black hole's mass starting at a radius of $0.3 \mathrm{pc}\left(7^{\prime \prime}\right)$ from $\mathrm{Sgr} \mathrm{A}^{*}$. At a radius of $1 \mathrm{pc}\left(24^{\prime \prime}\right)$ from $\mathrm{Sgr} \mathrm{A}^{*}$, near the tip of the minispiral seen in projection, the enclosed mass has nearly doubled, to about $8 \times 10^{6} M_{\odot}$. Thus, for a given circular orbit with a radius of $1 \mathrm{pc}$, the actual orbital velocity would be about 1.4 times higher than in a pure Keplerian approximation based on the mass of the black hole only. Apart from this effect, however, in several regions the radial velocities deviate even more significantly from those predicted from the Keplerian model.

\subsubsection{Non-Keplerian Components}

In the northwest end of the Eastern Arm near the location E17 (Figure 7(a)), a feature with radial velocities blueshifted up to $\sim-200 \mathrm{~km} \mathrm{~s}^{-1}$ shows a large deviation from the value of $\sim-100 \mathrm{~km} \mathrm{~s}^{-1}$ predicted by the Keplerian model (Figure 6). From Paumard et al. (2006), we find that at least five massive stars-IRS 34W (Ofpe/WN9), IRS 34E (O9-9.5I), IRS 34NW (WN7), IRS 7SW (WN8), and IRS 3E (WC5/6) - are located in a region with a radius of 2 '. 2 (indicated with a large black circle near region E16 in Figure 7(a)), referred to as IRS 34 hereafter. Except for IRS 3E, which has no proper motion measurements, the proper motion data of the remaining four stars (Paumard et al. 2006) show that the stars all move southwest toward the ionized flow with a mean transverse velocity of $185 \mathrm{~km} \mathrm{~s}^{-1}$ $\left(\right.$ P.A. $\left.=-134^{\circ}\right)($ as shown by an arrow in Figure $7(a))$. We compare the radial velocity of the stars with the spectrum (inset (c) in Figure 7(a)) of the $\mathrm{H} 30 \alpha$ line emission integrated from the IRS 34 region. The four stars besides IRS 3E show negative radial velocities in the range $-150 \mathrm{~km} \mathrm{~s}^{-1}$ to $-340 \mathrm{~km} \mathrm{~s}^{-1}$, which is blueshifted with respect to the peak velocity $\left(-146 \pm 4 \mathrm{~km} \mathrm{~s}^{-1}\right)$ of the broad $\left(\Delta V_{\mathrm{FWHM}}=160 \pm 8 \mathrm{~km} \mathrm{~s}^{-1}\right)$ spectral feature. The stellar winds from the Wolf-Rayet (WR) stars, evolved from early-type O-stars with mass-loss rate of $10^{-5} M_{\odot} \mathrm{yr}^{-1}$, appear to play a considerable role in the local kinematics. The strong stellar winds with velocities up to a few times $10^{3} \mathrm{~km} \mathrm{~s}^{-1}$ could sweep up the ionized flow of the Eastern Arm, causing the peculiar velocities and the velocity gradient across the Eastern Arm at the northwest end. In fact, the motions of the filaments associated with the minispiral found by Mužić et al. (2007) in infrared also suggest that a model of purely Keplerian motions 

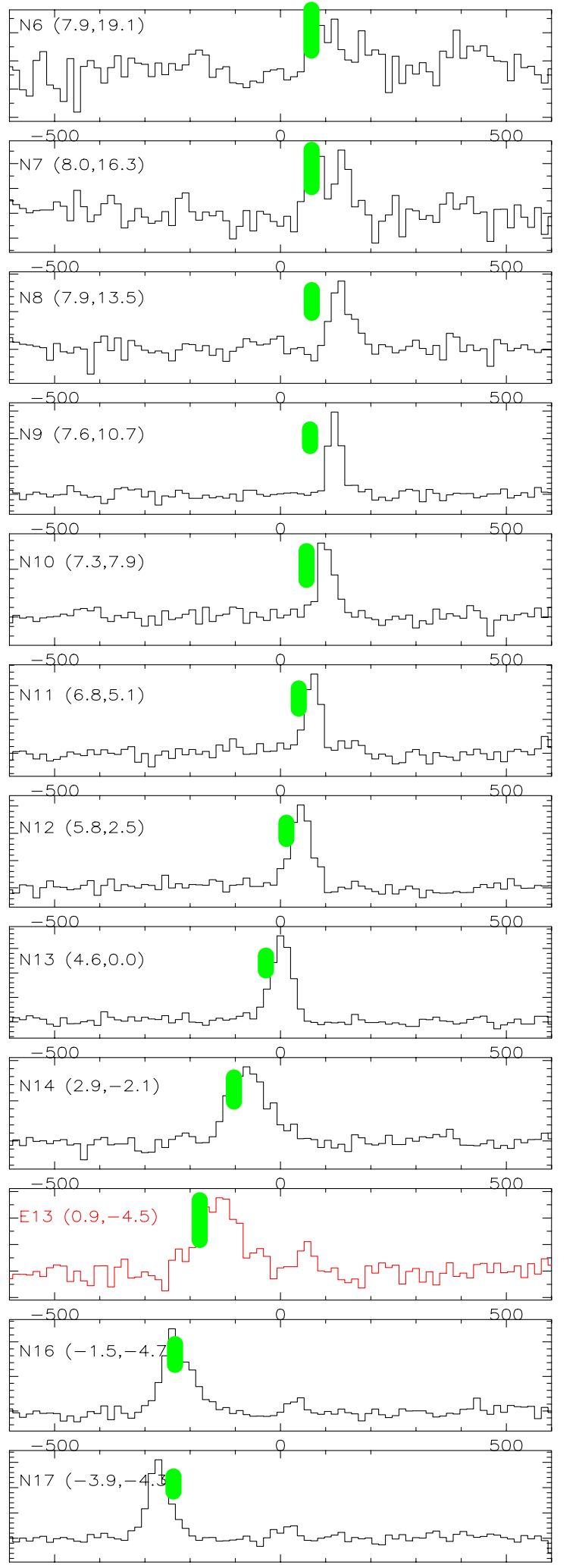

VIsr (km/s)
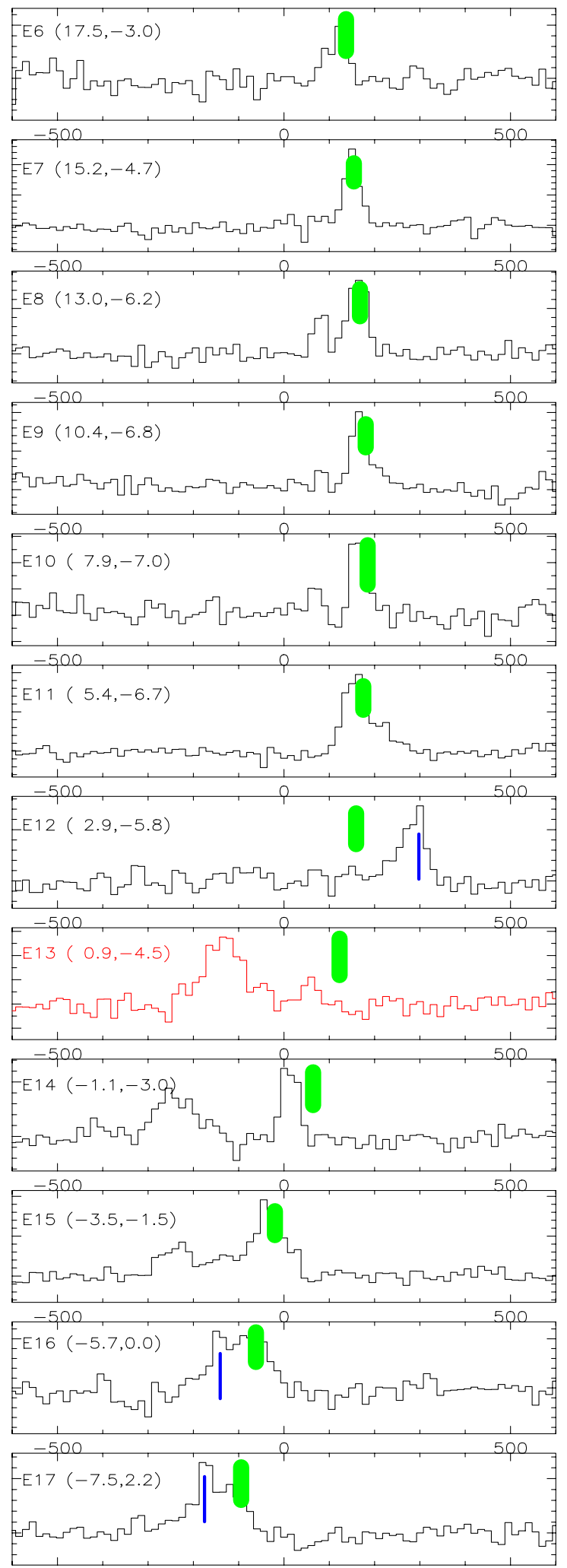

Vlsr $(\mathrm{km} / \mathrm{s})$

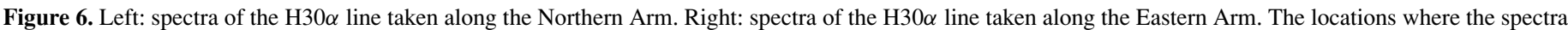

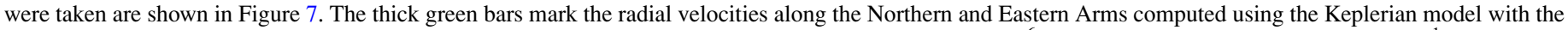

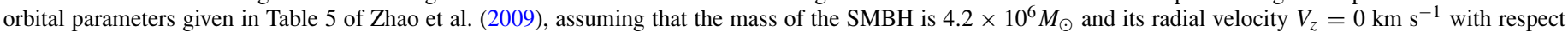

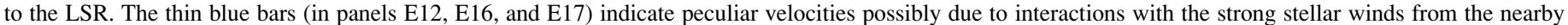

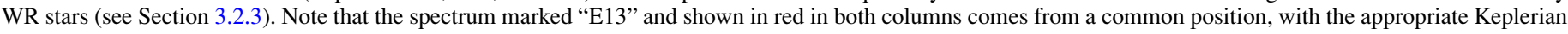
velocity marked for each arm.

(A color version of this figure is available in the online journal.) 

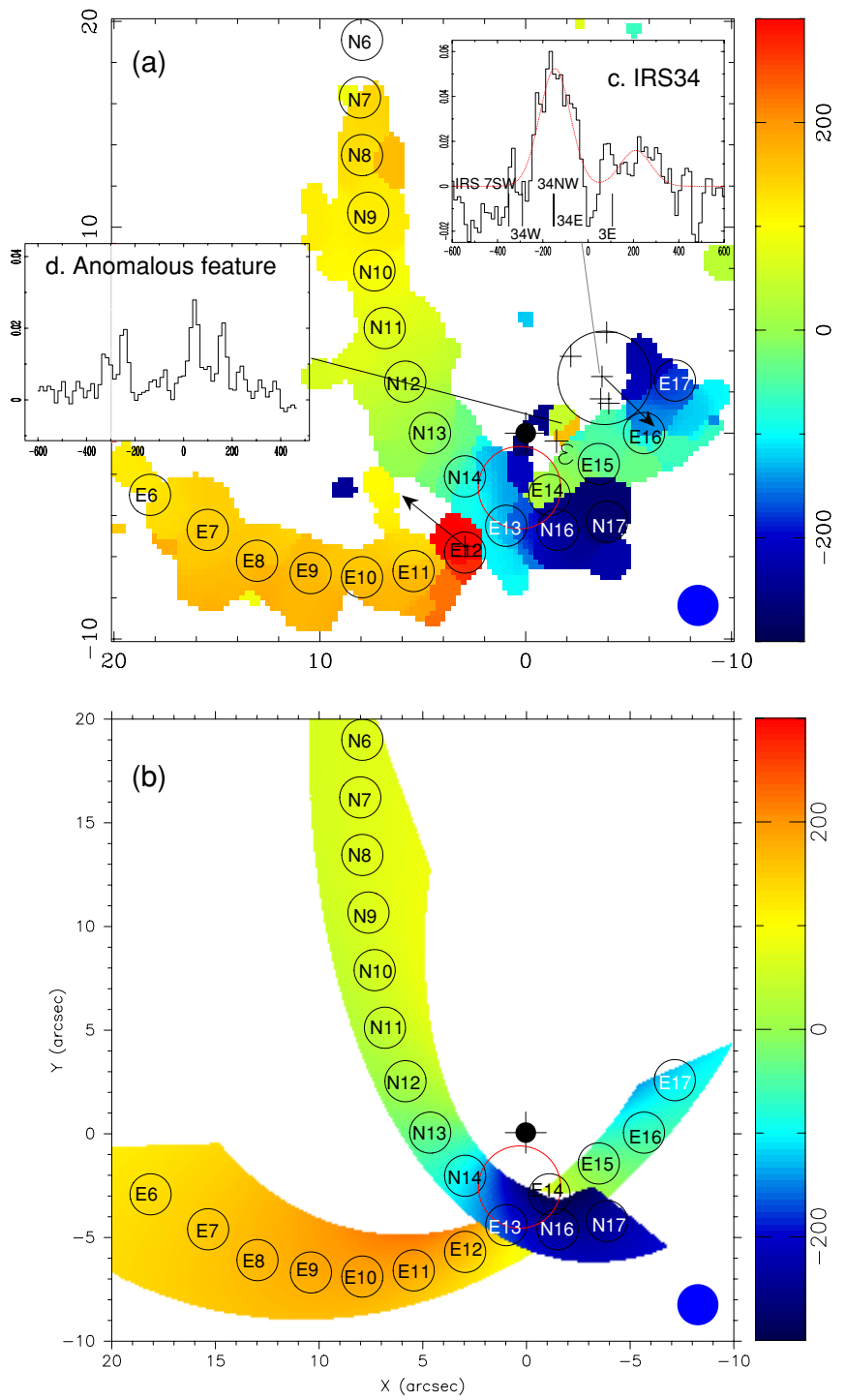

Figure 7. Comparison between the observed radial velocities (a) and those computed from the Keplerian model (b). The black circles along the two spiral arms mark the locations of the $\mathrm{H} 30 \alpha$ spectra displayed in Figure 6 . The red circle indicates the high temperature region. The dot-cross marks the position of $\mathrm{Sgr} \mathrm{A}^{*}$ at the coordinate origin. The inset (c) is the line spectrum of $\mathrm{H} 30 \alpha$ toward the IRS 34 region indicated with a large black circle, which includes the five massive stars IRS 7SW (WN8), IRS 34W (Ofpe/WN9), IRS 34E (O9-9.5I), IRS 34NW (WN7), and IRS 3E (WC5/6) (Paumard et al. 2006). The mean proper motion of the first four stars (and a fifth star without proper motion data) is indicated with an arrow near the E16 region in (a). The radial velocities of the five stars are marked with bars in inset (c). The proper motion of the IRS 9W star (WN8) from Paumard et al. (2006) is indicated with an arrow near the E12 region in (a). The inset (d) shows the $\mathrm{H} 30 \alpha$ line spectrum toward an anomalous feature $\left(-1^{\prime \prime} .5\right.$ and 0.5$)$ northwest of Sgr $\mathrm{A}^{*}$ and $1^{\prime \prime}$ north of $\varepsilon$.

(A color version of this figure is available in the online journal.)

of the gas is inadequate and the filaments could be due to the interaction of a fast wind with the minispiral.

In the tip region (Paumard et al. 2004) with peculiar radial velocity that deviates significantly from the Keplerian velocity by $+150 \mathrm{~km} \mathrm{~s}^{-1}$ (see panel (E12) in Figures 6 and 7(a) and (b)), there is also a WN8 star, IRS 9W. The radial velocity $\left(+140 \mathrm{~km} \mathrm{~s}^{-1}\right.$ ) of IRS 9W (Paumard et al. 2004) is similar to that of ionized gas in the Eastern Arm. A comparison between the stellar transverse velocity of $215 \mathrm{~km} \mathrm{~s}^{-1}\left(\right.$ P.A. $=51^{\circ}$ ) and that of $304 \mathrm{~km} \mathrm{~s}^{-1}$ (P.A. $=-67^{\circ}$ ) predicted from the Keplerian model for the ionized flow at the location of IRS 9W suggests that the star does not follow the orbit of the Eastern Arm but may run across the Eastern Arm. Then, the high-radial-velocity tip is likely to be the part of the gas in the ionized flow that is compressed and accelerated by the suspected strong wind from the IRS 9W star. The velocity discontinuity at the tip may be evidence for the presence of shocks in the region.

In addition, deviations from Keplerian motions are also observed in the Bar region. The non-Keplerian kinematics could be attributed both to the interaction between the ionized flows in the Northern and Eastern Arms and to the interaction of the ionized flows with the strong winds from the massive star clusters (the IRS 16 and IRS 13 clusters, for example). Wellorganized polarized emission from magnetically aligned dust grains in the central parsec has been observed (Aitken et al. 1991, 1998; Glasse et al. 2003), suggesting a magnetic field strength of $\geqslant 2 \mathrm{mG}$. The corresponding energy densities in the magnetic fields are $\sim 1.6 \times 10^{-7} \mathrm{erg}^{-3}$, about twice larger than the thermal energy densities in the ionized gas $\left(T_{e} \sim 7 \times 10^{3} \mathrm{~K}\right.$ and $n_{e} \sim 6 \times 10^{4} \mathrm{~cm}^{-3}$ at IRS $1 \mathrm{~W}$ ) but 2 orders of magnitude less than the kinetic energy densities of the orbital motions. The magnetic fields frozen in the ionized streamers cannot substantially alter the bulk motions of the gas but produce some cumulative magnetohydrodynamic (MHD) effects, such as orbital compression of the streamers as implied from the observed evidence for the convergence of field lines near Sgr A* (Glasse et al. 2003), and a helix of a natural morphology for a twisted magnetic field frozen into a plasma as observed in the Northern Arm (Yusef-Zadeh et al. 1989; Zhao et al. 2009). In the region close to Sgr A*, the magnetic field probably becomes stronger, and the MHD effects may also play a significant role in the kinematics of the ionized flows.

An interesting anomalous feature located $\sim 2^{\prime \prime}$ northwest of Sgr A* can be seen in the integrated $\mathrm{H} 30 \alpha$ line intensity image (Figure 1). The radial-velocity image (Figure 2) indicates a large velocity gradient across this feature. The $\mathrm{H} 30 \alpha$ line spectrum (inset (d) in Figure 7) suggests that this feature is composed of at least three spectral components $(\geqslant 4 \sigma)$ at radial velocities of $160 \mathrm{~km} \mathrm{~s}^{-1}, 39 \mathrm{~km} \mathrm{~s}^{-1}$, and $-250 \mathrm{~km} \mathrm{~s}^{-1}$, with a line width of $\sim 50 \mathrm{~km} \mathrm{~s}^{-1}$, a typical velocity width of the ionized gas in the minispiral arms. We note that there is a compact continuum source called $\varepsilon$ (Yusef-Zadeh \& Morris 1991), located 1" south of this anomalous feature, corresponding to a hole in the $\mathrm{H} 30 \alpha$ line images (e.g., Figures 1 and 7(a)). The source $\varepsilon$ appears to be moving away from Sgr A* with a transverse velocity of $340 \mathrm{~km} \mathrm{~s}^{-1}$ (Zhao et al. 2009), which has been interpreted as the confluence of gravitationally focused winds from the IRS 16 cluster (Wardle \& Yusef-Zadeh 1992). The H30 $\alpha$ line property of the anomalous feature appears to be consistent with the focused-wind model, suggesting that the velocity components in the anomalous feature correspond to ionized blobs of the minispiral arms that have been blown out of the region $\varepsilon$ due to the high pressure of the confluent stellar winds. However, given the closeness to Sgr A*, the detection of this feature needs to be further confirmed with higher-sensitivity observations.

Finally, in the locations with large angular offsets from Sgr A*, we noticed that the deviations in radial velocity from the Keplerian motions become significant for the Northern Arm $\left(>50 \mathrm{~km} \mathrm{~s}^{-1}\right)$, as shown in both Figures 6 and 7, while for the Eastern Arm, the deviations are less significant. The difference in the deviation of the observed radial velocities from the Keplerian velocities between the Northern and Eastern Arms suggests that the distribution of mass has a preference for the plane of the CND. 


\section{IONIZATION}

\subsection{The High-density Gas in the Northern and Eastern Arms}

We have shown that the $\mathrm{H} 30 \alpha$ lines observed with the SMA are excellent tracers of the high-density $\mathrm{H}$ II components in the Northern and Eastern Arms. Based on the observations and derived physical parameters, we can estimate the ionizing photon rate $\left(Q=n_{e}^{2} \alpha_{B} V\right)$ required for maintaining the ionizations in these regions for the case where the ionized gas is optically thick in the Lyman lines and where $\alpha_{B}$ is the ionization coefficient, and $V \approx \Omega D^{2} L f_{V}$ is the volume of the ionized clumps. The ionizing photon rate can be written as

$$
\begin{aligned}
Q(H)= & 1.31 \times 10^{45}\left[\frac{\alpha_{B}\left(T_{e}\right)}{\alpha_{B}\left(10^{4} \mathrm{~K}\right)}\right] \\
& \times\left[\frac{\theta_{B}}{\operatorname{arcsec}}\right]^{2}\left[\frac{n_{e}}{10^{4} \mathrm{~cm}^{-3}}\right]^{2}\left[\frac{D}{8 \mathrm{kpc}}\right]^{2} \\
& \times\left[\frac{L f_{V}}{10^{-3} \mathrm{pc}}\right] \text { photons s}^{-1},
\end{aligned}
$$

where $\theta_{B}$ is the resolution of the observations (beam) with the assumption that source fills the beam and $\alpha_{B}\left(T_{e}\right)$, the total recombination coefficient to excited levels, is given by Hummer $\&$ Seaton (1963). We calculated the values of the inferred ionizing photon flux required to maintain the ionization of the high-density components in the Northern and Eastern Arms as well as the $\mathrm{H}$ II mass $\left(M_{\mathrm{H} \text { II }}\right)$ and excitation parameter $(U)$. On the assumption that the $\mathrm{H}$ II gas is internally ionized, the equivalent zero-age main-sequence (ZAMS) star that is the ionizing source was estimated. The internal-ionization model requires at least $\sim 25$ O9-type stars embedded and roughly evenly distributed in both the Northern and Eastern Arms.

\subsection{Ionizing Sources}

We now examine where the known OB stars lie in the region. Figure 8 shows the 90 massive stars within a radius of $14^{\prime \prime}$ (0.5 pc) of Sgr A* identified by Paumard et al. (2006), among which 55 are $\mathrm{OB}$ stars. The $14 \mathrm{OB}$ stars within a radius of $0.85(0.03 \mathrm{pc})$ from $\mathrm{Sgr} \mathrm{A}^{*}$ are known as S stars. No obvious correlations appear between the location of 41 OB stars outside the radius of $0^{\prime \prime} .85$ and the distribution of the high-density $\mathrm{H}$ II gas in the minispiral arms on the projected plane. If we assume the average solid angle for the minispiral subtended to be $\Omega_{s}$, then the required number of ionizing photons from the stars will be

$$
Q^{*}(H)=Q(H)\left(4 \pi / \Omega_{s}\right) .
$$

If we assume $4 \pi / \Omega_{s} \sim 10$, then 250 O9-type stars are required.

For the lower-density $\left(\bar{n}_{e} \sim 8 \times 10^{2} \mathrm{~cm}^{-3}\right)$ component $\mathrm{B}$, the ionized mass of $\sim 340 M_{\odot}$ is inferred, requiring a Lyman photon flux of $1.2 \times 10^{50}$ photons s $^{-1}$ or $\sim 100$ O9-type stars (ZAMS). The 250 O9-type stars required for externally maintaining the ionization of the minispiral appear to be adequate in supplying the leaking Lyman photons from the minispiral to further ionize the lower-density component that pervasively distributes in the region. Thus, the ionizing source to maintain the ionization state of Sgr A West is equivalent to 250 O9-type stars (ZAMS). Considering that a substantial amount of the ionizing photons can be produced by the hot evolved massive stars and the survey of Paumard et al. (2006) is incomplete, there may be enough stars to ionize the region.

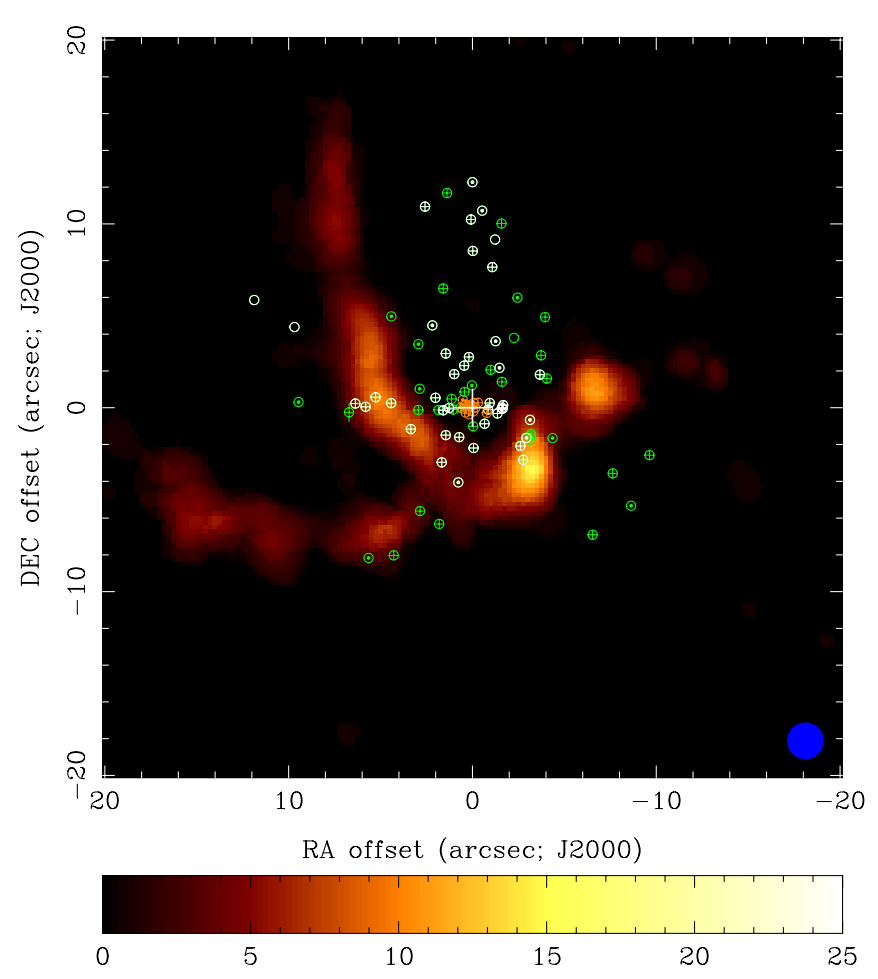

Figure 8. Ninety massive stars of Paumard et al. (2006) are overlaid on the $\mathrm{H} 30 \alpha$ line image. Among the 90 massive stars, 14 OB stars (orange), known as $\mathrm{S}$ stars, are located within a radius of 0.85 from Sgr A* (white plus sign), 41 OB stars (white) are outside the S cluster, and the rest are Ofpe/WN9 WR stars (green). The cross- and dot- circles represent the stars with proper motion data, and the open circles indicate stars with no proper motion data.

(A color version of this figure is available in the online journal.)

The origin of the ionized minispiral arms and the birthplace of the massive stars found in the central parsec are still matters of conjecture. There are two possible models that may explain the presence of the massive stars near the SMBH. First, the stars formed elsewhere outside the central parsec and migrated to the Galactic center (Gerhard et al. 2001). However, compared with the age of the massive stars in the central parsec, it takes too long for stars to move to the center from several parsecs away by losing their orbital energy through dynamical friction (Portegies Zwart et al. 2003; Kim \& Morris 2003). Alternatively, the stars may have formed near the $\mathrm{SMBH}$ by an unusual astrophysical process, such as the fragmentation of a massive disk rotating near the SMBH (Nayakshin et al. 2007). Recent modeling and numerical simulations (Nayakshin et al. 2007; Wardle \& Yusef-Zadeh 2008; Bonnell \& Rice 2008) show that for an infalling giant molecular cloud $\left(10^{4-5} M_{\odot}\right)$ interacting with an SMBH $\left(1-3 \times 10^{6} M_{\odot}\right)$, a small fraction of the gas cloud becomes bounded to the black hole, forming an eccentric disk that quickly fragments to form stars. The tidal disruption of the self-gravitating clouds formed coherent spiral structures, i.e., the minispiral arms, which are ionized by the Lyman continuum photons produced from the newly formed massive stars. Further evidence for the existence of two counter-rotating stellar disks is given in Bartko et al. (2009). New observations of the young starburst cluster in the Galactic center (Bartko et al. 2010) are consistent with the top-heavy initial mass function (IMF) proposed by Bonnell \& Rice (2008).

\section{SUMMARY AND CONCLUSION}

We report results from the SMA observations of the $\mathrm{H} 30 \alpha$ line emission from the Galactic center at a resolution of $2^{\prime \prime}$. 
We measured the velocity profiles over the central $60^{\prime \prime}(2 \mathrm{pc})$ region around $\mathrm{Sgr} \mathrm{A}^{*}$. We carried out an analysis of the line and continuum data based on an isothermal homogeneous $\mathrm{H}$ II model for each component. With the constraints from the SMA H3O $\alpha$ data at $1.3 \mathrm{~mm}$ and the VLA H92 $\alpha$ data at $3.6 \mathrm{~cm}$ and continuum data at $1.3 \mathrm{~cm}$, we determined the physical conditions of ionized gas in the Northern and Eastern Arms of the minispiral. We found that the typical electron density and kinetic temperature in the arms are 3-21 $\times 10^{4} \mathrm{~cm}^{-3}$ and $5000-13,000 \mathrm{~K}$, respectively. The highest density of $2.1 \times 10^{5} \mathrm{~cm}^{-3}$ occurs in the IRS 13 region. Higher temperatures up to $13,000 \mathrm{~K}$ are found in the Bar region. Both the $\mathrm{H} 30 \alpha$ and $\mathrm{H} 92 \alpha$ line profiles are broadened due to large velocity gradients along the line of sight and across the $2^{\prime \prime}$ beam produced by the dynamical motions near Sgr A*. For the H92 $\alpha$ line, the line width due to pressure broadening appears to be comparable to that of thermal Doppler broadening, while for the $\mathrm{H} 30 \alpha$ lines, the pressure broadening is negligible.

Using the orbital parameters derived under the assumption that the three minispiral flows are in Keplerian motion, we calculated the three-dimensional geometry of the minispiral structure. We showed that the ionized flows of the Northern Arm and Western Arc are almost coplanar, and the plane of the ionized flow in the Eastern Arm is nearly perpendicular to that of the Northern Arm and Western Arc. The Eastern Arm flow collides with the Northern Arm flow in the Bar region located $0.1-0.2$ pc south of and behind Sgr A*. The high-kinetictemperature gas in the Bar is probably due to the heat in shocks generated by collisions.

We compared observed radial velocities in the Northern and Eastern Arms with those computed from the Keplerian model with a mass of $4.2 \times 10^{6} M_{\odot}$ of the SMBH at the position of Sgr A*. We show that Keplerian motion dominates the kinematics of the minispiral arms. In some regions, significant deviations exist between the observed radial velocities and those computed from the Keplerian model. Near the IRS 9W and IRS 34 regions, the observed peculiar velocities in the minispiral are likely a result of the ionized gas being swept up by the strong stellar winds from nearby WR stars.

A total Lyman continuum flux of $3 \times 10^{50}$ photons s$^{-1}$ is needed to maintain the ionization of the gas in Sgr A West with an inferred ionized mass of $350 M_{\odot}$, which requires $\sim 250$ O9-type (ZAMS) stars. About 10\% of the total number of UV photons is required to externally maintain the ionization of the high-density gas in the Northern and Eastern Arms, which have an ionized mass of $12 M_{\odot}$, less than $5 \%$ of the total ionized mass (Liszt 2003).

We are grateful to Carolann Barrett for her careful editing of our manuscript. We also thank Harvey Liszt for discussing aspects of his 2003 Keplerian model and for encouraging us to make these SMA observations. The research has made use of NASA's Astrophysics Data System. Support for D.P.M. was provided by NASA through Hubble Fellowship grant HST-HF-51259.01 awarded by the Space Telescope Science Institute, which is operated by the Association of Universities for Research in Astronomy, Inc., for NASA, under contract NAS $5-26555$.

\section{REFERENCES}

Aitken, D. K., Gezari, D., Smith, C. H., McCaughrean, M., \& Roche, P. F. 1991, ApJ, 380, 419
Aitken, D. K., Smith, C. H., Moore, T. J. T., \& Roche, P. F. 1998, MNRAS, 299 743

Anantharamaiah, K. R., Viallefond, F., Mohan, N. R., Goss, W. M., \& Zhao, J.-H. 2000, ApJ, 537, 613

Anantharamaiah, K. R., Zhao, J.-H., Goss, W. M., \& Viallefond, F. 1993, ApJ, 410,110

Bartko, H., et al. 2009, ApJ, 697, 1741

Bartko, H., et al. 2010, ApJ, 708, 834

Bonnell, L. A., \& Rice, W. K. M. 2008, Science, 321, 1060

Briggs, D. S. 1995, PhD thesis, NMIMT

Brocklehurst, M., \& Leeman, S. 1971, ApL, 9, 35

Buchholz, R. M., Schödel, R, \& Eckart, A. 2009, A\&A, 499, 483

Ekers, R. D., van Gorkom, J. H., Schwarz, U. J., \& Goss, W. M. 1983, A\&A, 122,143

Gerhard, O., Kronawitter, A., Saglia, R. P., \& Bender, R. 2001, AJ, 121, 1936

Ghez, A. M., et al. 2008, ApJ, 689, 1044

Gillessen, S., Eisenhauer, F., Trippe, S., Alexander, T., Genzel, R., Martins, F., \& Ott, T. 2009, ApJ, 692, 1075

Glasse, A. C. H., Aitken, D. K., \& Roche, P. F. 2003, Astron. Nachr., 324, 563

Gordon, M. A., \& Sorochenko, R. L. 2002, Radio Recombination Lines. Their Physics and Astronomical Applications (Dordrecht: Kluwer)

Güsten, R., Genzel, R., Wright, M. C. H., Jaffe, D. T., Stutzki, J., \& Harris, A. I. 1987, ApJ, 318, 124

Hansen, B. M. S., \& Milosavljevic, M. 2003, ApJ, 593, L77

Hummer, D. G., \& Seaton, M. J. 1963, MNRAS, 125, 437

Kim, S. S., \& Morris, M. 2003, ApJ, 597, 312

Lacy, J. H., Achtermann, J. M., \& Serabyn, E. 1991, ApJ, 380, L71

Liszt, H. S. 2003, A\&A, 408, 1009

Loeb, A. 2004, MNRAS, 350, 725

Lutz, D., Krabbe, A., \& Genzel, R. 1993, ApJ, 418, L244

Melia, F, Coker, R. F., \& Yusef-Zadeh, F. 1996, ApJ, 460, L33

Mezger, P. G., \& Henderson, A. P. 1967, ApJ, 147, 471

Mužić, K., Eckart, A., Schödel, R., Meyer, L., \& Zensus, A. 2007, A\&A, 469, 993

Nayakshin, S., Guadra, J., \& Springel, V. 2007, MNRAS, 379, 21

Oh, S., Kim, S. S., \& Figer, D. F. 2009, J. Korean Astron. Soc., 42, 17

Paumard, T., Maillard, J. P., \& Morris, M. 2004, A\&A, 426, 81

Paumard, T., et al. 2006, ApJ, 643, 1011

Phookun, B., Anantharamaiah, K. R., \& Goss, W. M. 1998, MNRAS, 295, 156

Portegies Zwart, S. F., McMillan, S. L. W., \& Gerhard, O. 2003, ApJ, 593, 352

Puxley, P. J., Brand, P. W. J. L., Moore, T. J. T., Mountain, C. M., \& Nakai, N. 1991, MNRAS, 248, 585

Roberts, D. A., \& Goss, W. M. 1993, ApJS, 86, 133

Roberts, D. A., Yusef-Zadeh, F., \& Goss, W. M. 1996, ApJ, 459, 627

Rodriguez-Rico, C. A., Viallefond, F., Zhao, J.-H., Goss, W. M., \& Anantharamaiah, K. R. 2004, ApJ, 616, 783

Sanders, R. H. 1998, MNRAS, 294, 35

Sault, B., Teuben, P., \& Wright, M. 1995, in ASP Conf. 77, ADASS IV, ed. R. Shaw, H. E. Payne, \& J. J. E. Hayes (San Francisco, CA: ASP), 433

Schödel, R., Najarro, F., Mužić, K., \& Eckart, A. 2010, A\&A, 511, A18

Schwarz, U. J., Bregman, J. D., \& van Gorkom, J. H. 1989, A\&A, 215, 33

Scoville, N. Z., Stolovy, S. R., Rieke, M., Christopher, M., \& Yusef-Zadeh, F. 2003, ApJ, 594, 294

Shaver, P. A. 1975, Pramana, 5, 1

Vollmer, B., \& Duschl, W. J. 2000, New Astron., 4, 581

Walmsley, C. M. 1990, A\&AS, 82, 201

Wardle, M., \& Yusef-Zadeh, F. 1992, Nature, 357, 308

Wardle, M., \& Yusef-Zadeh, F. 2008, ApJ, 683, 37

Wilson, T. L., Rohlfs, K., \& Hüttemeister, S. 2009, Tools of Radio Astronomy (5th ed.; Berlin: Springer), 369

Yusef-Zadeh, F., \& Morris, M. 1991, ApJ, 371, L59

Yuzef-Zadeh, F., Morris, M., \& Ekers, R. 1989, in IAU Symp. 136, The Center of the Galaxy, ed. M. Morris (Norwell: Kluwer), 443

Yusef-Zadeh, F., Roberts, D. A., \& Biretta, J. 1998, ApJ, 499, L159

Zhao, J.-H., Anantharamaiah, K. R., Goss, W. M., \& Viallefond, F. 1996, ApJ, 472,54

Zhao, J.-H., Anantharamaiah, K. R., Goss, W. M., \& Viallefond, F. 1997, ApJ, 482,186

Zhao, J.-H., Morris, M. R., Goss, W. M., \& An, T. 2009, ApJ, 699, 186 